\title{
Strukturen von Wohnungsleerstand in Deutschland. Eine Analyse der Gebäude- und Wohnungszählung 2011 in deutschen Gemeinden
}

\section{Structure of housing vacancy in Germany. A Typology of Attributes of the German Housing Census 2011 in German Municipalities}

https://doi.org/10.2478/rara-2019-0011

Eingegangen: 28. Juni 2018; Angenommen: 29. Januar 2019

Zusammenfassung: Die Leerstandsquote ist ein zentraler Indikator der Wohnungsmarktbeobachtung und wird zur Beurteilung von Wohnungsmarktsituationen herangezogen. In Deutschland variieren die Leerstandsquoten der Städte und Gemeinden stark, was unter anderem durch die Merkmale unterschiedlicher Bestände des Wohnungsmarktes bedingt ist, wie z. B. Baualter oder Wohnungsgröße. Für die Abbildung der gesamten dabei auftretenden Variationen wurde bislang kaum eine systematische Analyse von Leerstandsstrukturen erstellt. Vor diesem Hintergrund wird im vorliegenden Beitrag das Ziel verfolgt, die differenzierte Struktur des Leerstandes für deutsche Gemeinden aufzuzeigen. Dafür werden Daten der Gebäude- und Wohnungszählung (GWZ) 2011 verwendet, die flächendeckend und kleinräumig Leerstand differenziert nach 50 Ausprägungen erfassten. Diese Ausprägungen werden auf ihren Erklärungsgehalt für die Leerstandsquote geprüft. Darauf aufbauend werden räumliche Leerstandstypen gebildet, die die Leerstandsstruktur in Deutschland auf kommunaler Ebene wiedergeben. Der Beitrag macht deutlich, dass in erster Linie Merkmale des Baualters sowie der Gebäude- und Wohnungsgröße die Leerstandsquote erklären, sich aber deutliche Unterschiede zwischen Ost- und Westdeutschland etwa in Bezug auf die Eigentumsform zeigen. Die kommunale Typisierung schärft und verräumlicht diese Erkenntnisse und unterstreicht, dass neben Ost-West- sowie Stadt-Land-Unterschieden auch Typen der Leerstandsstruktur existieren, die im gesamten Bundesgebiet vorkommen. Der Beitrag stellt eine Analyse vor, die mithilfe der entwickelten Typologie helfen soll, Leerstand zu differenzieren, zu bewerten und einzuordnen. Daraus lassen sich dann nicht nur Aussagen zum Einsatz von städtebaulichen und wohnungspolitischen Maßnahmen ableiten, auch die Raum- bzw. Wohnungsmarktbeobachtung auf Bundesebene lässt sich weiter qualifizieren.

Schlüsselwörter: Wohnungsleerstand, Leerstandsstruktur, Wohnungsmarktbeobachtung, Gebäude- und Wohnungszählung (GWZ), Gemeindetypisierung

\footnotetext{
*Corresponding author: Dr. Manuel Wolff, Humboldt Universität zu Berlin, Geographisches Institut, Unter den Linden 6, 10099 Berlin, Deutschland, E-mail: manuel.wolff@ hu-berlin.de

Prof. Dr. Dieter Rink: Helmholtz-Zentrum für Umweltforschung - UFZ, Department Stadt- und Umweltsoziologie, Permoserstraße 15, 04318 Leipzig, Deutschland
} 
Abstract: The vacancy rate is a key indicator of housing market monitoring and is used to assess housing market situations. In Germany, the vacancy rates of cities and rural areas vary widely, which is due to the different characteristics of the housing stock e.g. in terms of building age or flat size. Up to now, there are hardly systematic analyses of the vacancy structure which capture the full variation of vacancy patterns. Against this background the paper aims at detecting the different structure of vacancies for German municipalities. Therefore, small-scale data from the Building and Housing Census (GWZ) 2011 is used, covering the whole country. The vacancy data can be differentiated according to 50 characteristics which are checked against their explanatory power for the vacancy rate. Based on this, spatial vacancy types are built that reflect the vacancy structure in Germany at the municipal level. The paper underlines that building age as well as building and flat size have the highest explanation power. However, there are clear differences between Eastern and Western Germany, for example with regard to ownership. The typology of municipalities sharpens and spatially locates these findings. In addition to east-west and urban-rural differences there are types of vacancy structure that occur throughout Germany. The paper presents an analysis which helps to differentiate, evaluate and classify vacancy structures. The results allow to derive evidences for urban planning or housing policy measures as well as to qualify the housing monitoring at the federal level.

Keywords: Housing vacancy, Vacancy structure, Housing market monitoring, Building and housing census (GWZ), Typology of municipalities

\section{Einleitung}

Die Leerstandsquote wird als zentraler Indikator der Wohnungsmarktbeobachtung in Deutschland zur Beurteilung unterschiedlicher Wohnungsmarktsituationen herangezogen. Daraus können z. B. allgemeine Aussagen über die Funktionsfähigkeit von Wohnungsmärkten abgeleitet und insbesondere abgeschätzt werden, ob diese entspannt, angespannt oder gar krisenhaft sind. So indizieren etwa niedrige Leerstandsquoten Wohnungsknappheit bzw. Wohnungsnot für Mieter, aber eine entspannte Situation für Vermieter; bei hohen Leerstandsquoten verhält es sich genau umgekehrt. In einer ersten Auswertung der Leerstandsdaten der Gebäude- und Wohnungszählung (GWZ) 2011 haben Rink und Wolff (2015) diesbezüglich eine Heuristik für die Einteilung von Leerstandsquoten vorgeschlagen. In Deutschland variieren die Leerstandsquoten stark und zeigen damit sowohl (sehr) angespannte als auch (sehr) entspannte Wohnungsmärkte an (Rink/Wolff 2015: 320). Eine wesentliche Erklärung dafür ist, dass unterschiedliche Bestände des Wohnungsmarktes von Leerstand betroffen sind und damit Wohnungen mit bestimmten Merkmalen unterschiedliche Leerstandsquoten aufweisen (vgl. Voigtländer/Demary/Gans et al. 2009). So können Leerstände etwa vom Baualter, der Gebäudegröße sowie dem Gebäudezustand abhängen, sie variieren in Abhängigkeit der Wohnungsgröße, der Raumanzahl oder der Ausstattung. Darüber hinaus können auch bestimmte Lagekriterien eine Rolle spielen wie Lärmbelastung, Image oder Infrastrukturausstattung (Glatter 2003: 161). Ursache kann aber auch die Markt- fähigkeit einzelner Immobilien in Bezug auf die jeweilige Nachfrage sein. Schließlich variiert der Leerstand auch in Relation auf die jeweils gewählte räumliche Skala, wie dies Gutting und Vandrei (2018: 11) für Hessen und Rheinland-Pfalz demonstriert haben.

Die Leerstandsquote ist aber auch davon abhängig, was als Leerstand zählt. Bislang existiert in der Wissenschaft jedoch keine allgemein anerkannte Definition von Wohnungsleerstand. Es lassen sich enge von weiten Definitionen unterscheiden. In der Immobilienökonomie wird etwa "von Leerstand von Wohnungen gesprochen, wenn diese auf dem Wohnungsmarkt angeboten werden und nach mindestens drei Monaten noch keine (neuen) Mieter gefunden worden sind“" (Klebsch 1997: 536). In dieser engen Definition werden nur die leerstehenden Wohnungen einbezogen, die auf dem Markt angeboten werden und die nicht Teil der normalen Fluktuation sind (Leerstand bis drei Monate). Zum Abriss vorgesehene oder von Umbau bzw. Modernisierung betroffene Wohnungen zählen ebenso wenig dazu (Klebsch 1997). Eine solche Definition produziert entsprechend auch eine geringe(re) Leerstandsquote. In weiten Definitionen, wie sie insbesondere in Städtebau und Planung verwendet werden, „bezeichnet Leerstand (ganz allgemein betrachtet) nutzbare Flächen in Gebäuden, die zur Zeit nicht genutzt werden“" (BMVBS/BBR 2007: 23). Mit dieser Definition gelangt man zu einer höheren Leerstandsquote, da jeglicher Leerstand gezählt wird, allerdings ist nicht klar definiert, was „Nutzung" heißt. Im vorliegenden Beitrag wird eine statistische Definition verwendet, die für Erhebungszwecke entwickelt wurde und die der Gebäude- und Wohnungszählung (GWZ) von 2011 
zugrunde lag (vgl. Kapitel 3). Diese lässt sich zwischen engen und weiten Definitionen verorten, sie zielt vor allem auf eine eindeutige methodische Erfassung ab.

Um die Leerstandsquote als wichtige Messgröße und Grundlage etwa für wohnungspolitische Entscheidungen zu qualifizieren, bedarf es neben der Quantifizierung der Leerstandsquote insbesondere einer weiterführenden systematischen Analyse von Leerstandsmerkmalen und Leerstandsstrukturen. Für die Abbildung der gesamten dabei auftretenden Variationen muss auf Daten zurückgegriffen werden, die entsprechende Informationen enthalten sowie flächendeckend verfügbar sind. Für Deutschland sind solche Daten erstmals im Zuge der Gebäude- und Wohnungszählung (GWZ) 2011 erhoben worden. Dabei wurden Merkmale von Gebäuden als auch von Wohnungen erfasst - etwa in Bezug auf das Baualter, die Gebäudegröße, die Heizungsart, die Eigentumsform, die Wohnungsgröße und -fläche, die sanitäre Ausstattung sowie die Wohnungsnutzung im Sinne von leerstehend oder nicht. Nach unserem Kenntnisstand wurde jedoch noch keine systematische Analyse der Leerstandsstruktur unter Verwendung dieser methodisch einheitlichen Vollerhebung durchgeführt, die es erlaubt, sowohl flächendeckende als auch kleinräumige Aussagen zu treffen als auch den Indikator Leerstandsquote für eine weiterführende wohnungspolitische Diskussion auf Bundesebene zu qualifizieren.

Vor diesem Hintergrund wird in diesem Beitrag das Ziel verfolgt, die differenzierte Struktur des Leerstandes für deutsche Gemeinden aufzuzeigen, indem einzelne Leerstandsmerkmale der Gebäude- und Wohnungszählung 2011 auf ihren Erklärungsgehalt für die Leerstandsquote getestet sowie räumliche Leerstandstypen anhand von Merkmalskombinationen identifiziert werden. Dadurch wird eine differenzierte Bewertung der Leerstandsstruktur möglich, die eine Grundlage für Leerstandsprognosen bilden kann (vgl. BMVBS/BBR 2007: 145). Der Beitrag fragt vor diesem Hintergrund:

- Welche Merkmale haben welchen Einfluss auf die Leerstandsquote?

- Welche Leerstandsstrukturen existieren auf Gemeindeebene?

- Inwieweit spiegeln die Leerstandsstrukturen die Wohnungsbestandsstrukturen wider?

Der Beitrag reflektiert in Kapitel 2 zunächst den bisherigen Forschungsstand und stellt in Kapitel 3 den verwendeten Datensatz sowie die Analysemethodik vor. Inwieweit einzelne Merkmalsausprägungen einen Erklärungsgehalt für die jeweilige Leerstandsquote haben und welche Typen der Leerstandsstruktur sich für Gemeinden abbilden lassen, wird in Kapitel 4 dargestellt. In Kapitel 5 werden dann der Analyseansatz sowie die Datengrundlage kritisch betrachtet und in einem breiteren methodischen und wissenschaftlichen Kontext diskutiert. Der Beitrag schließt mit einem Ausblick zur Verwendung der Analyseergebnisse. ${ }^{1}$

\section{Ursachen und Struktur von Leerständen}

In der Literatur zum Thema Wohnungsleerstand sind die Zusammenhänge zwischen der Leerstandshöhe, genauer der Leerstandsquote, und den Merkmalen der betroffenen Bestände sowie der Wohnungsmärkte bislang nicht systematisch analysiert worden (Henger/ Schier/Voigtländer 2013; BBSR 2014). Freilich finden sich in den einschlägigen Publikationen zum Thema viele Hinweise, die jedoch im Folgenden nicht im Einzelnen aufgelistet werden können. Vielmehr sollen einige übergreifende Gesichtspunkte herausgearbeitet werden. Die Höhe des Leerstandes in Wohnungsmärkten ist zunächst im Wesentlichen von grundlegenden Prozessen auf der Angebots- und Nachfrageseite abhängig (BBSR 2011: 75). Dabei wird in der Regel auf zwei Prozesse bzw. Gründe abgehoben: eine mehr oder weniger rasche bzw. massive Ausweitung des Angebots (Angebotsausweitung) und ein mehr oder weniger starker Rückgang bzw. eine Schrumpfung der Nachfrage (Nachfragerückgang). Hier wird außerdem noch ein längerfristiges bzw. dauerhaftes Desinvestment in die Wohnungsbestände als Prozess bzw. Grund angeführt (vgl. auch Rink/Wolff/Diez 2017).

Bei einer Angebotsausweitung wird das Wohnungsangebot bei mehr oder weniger gleichbleibender Nachfrage deutlich über den bestehenden Bedarf ausgeweitet. Leerstand tritt hier als Überangebot bzw. Überhang auf dem Wohnungsmarkt zutage. Hier sind es vor allem neugebaute Wohnungen oder Häuser, die leer stehen, da es keine ausreichende Nachfrage gibt. Leerstand infolge Angebotsausweitung tritt typischerweise auch als Folge von Spekulation auf, wie etwa im Zuge von Immobilienblasen, wie wir sie vor einigen Jahren in Europa etwa

1 Der Beitrag ist das Ergebnis des vom Bundesinstitut für Bau-, Stadt- und Raumforschung (BBSR) geförderten Forschungsprojektes „Auswertung von Leerstandsmerkmalen der Gebäude- und Wohnungszählung (GWZ) 2011“. Wir bedanken uns bei den Kollegen des BBSR Matthias Waltersbacher, Eva Korinke und Alexander Schürt für die produktive Zusammenarbeit. 
in Spanien oder Irland erlebt haben (Hoekstra/VakiliZad 2011; Kitchin/O'Callaghan/Gleeson 2014), wodurch auch in großem Ausmaß neu errichtete Gebäude vom Leerstand betroffen waren. Leerstand infolge Angebotsausweitung kann aber auch die (unbeabsichtigte) Folge politischer Regulation durch spezielle Steuergesetze und Subventionen, aber auch der Wohnungs-, Boden-, Stadterneuerungs- und Baupolitik sein. Ein instruktives Beispiel dafür ist Ostdeutschland, wo in den 1990er-Jahren zahlreiche Programme angewandt und Instrumente eingesetzt wurden, um die aus der DDR überkommene Wohnungsnot zu beheben. Insbesondere die Möglichkeit, bei Neubauten oder Sanierungen bis zu $50 \%$ der Summe steuerlich abschreiben zu können (Sonder-AfA), mobilisierte zahlreiche Investitionen und führte zu einem Bau- und Sanierungsboom. Dieser hatte dann Ende der 1990er-/Anfang der 2000er-Jahre einen massiven Leerstand insbesondere bei sanierten, teilweise auch bei neugebauten Wohnungen zur Folge (Pfeiffer/Simons/ Porsch 2001). Derzeit ist - allerdings auf einem niedrigeren Niveau - eine solche Angebotsausweitung in den ländlichen Räumen und Kleinstädten Deutschlands als Folge von Niedrigzinsen zu beobachten (Deschermeier/Henger/Seipelt et al. 2017: 3), selbst in Regionen, für welche sinkende Haushaltszahlen prognostiziert werden (vgl. Held/Waltersbacher 2015). Schließlich ist die angebotsseitige Ausweitung des Leerstands teilweise bedingt bzw. verschärft durch den Trägheitseffekt von Wohnungsmärkten. Damit ist gemeint, dass auf den Wohnungsbedarf mit zeitlicher Verzögerung reagiert wird, da erst Bauland erworben, planerische Verfahren durchlaufen und Genehmigungen eingeholt werden müssen, ehe der Bau starten kann. Neue oder sanierte Wohnungen kommen so teilweise erst nach Jahren auf den Markt, wenn der Bedarf bereits wieder gesättigt sein kann (Vaché 2016: 90). Ein anschauliches Beispiel für diesen Trägheitseffekt ist die sogenannte Wohnungshalde, die in der Bundesrepublik Mitte der 1970er-Jahren im Gefolge eines Baubooms entstanden war. Diesem hatte Anfang der 1970er-Jahre die Diagnose einer Wohnungsknappheit zugrunde gelegen, die jedoch zu dem Zeitpunkt, als die meisten neuen Wohnungen auf den Markt kamen, schon nicht mehr gegeben war (Kreibich 1985: 183).

Beim Nachfragerückgang verringert sich die Nachfrage, während das Angebot mehr oder weniger gleich bleibt, insbesondere hinsichtlich der Quantität. Die Ursachen für den Rückgang können beispielsweise Fernwanderung und Suburbanisierung sein, längerfristig etwa der demographische Wandel. Auch hierfür ist Ostdeutschland ein gutes Beispiel, bedingt durch großräumige
Schrumpfungsprozesse ist hier Wohnungsleerstand zu einem strukturellen Merkmal der Wohnungsmärkte geworden (Rink/Wolff/Diez 2017). Der Nachfragerückgang kann auch abrupt erfolgen, etwa ausgelöst durch Wirtschaftskrisen, Konflikte und Kriege oder Epidemien. So können etwa gewalttätige Konflikte, Kriege und Bürgerkriege, aber auch Epidemien, Fluchtwellen auslösen, wo selbst große Städte verlassen werden und zu großen Teilen leer stehen. Wirtschafts- und Finanzkrisen können ebenfalls innerhalb relativ kurzer Zeit zu Abwanderungswellen führen. Ein anschauliches Beispiel für einen derartigen Nachfragerückgang ist Spanien, als nach dem Platzen der Immobilienblase und dem Einsetzen der Finanz- und Wirtschaftskrise 2008 viele junge Spanier das Land verließen. (Hoekstra/Vakili-Zad 2011). Derartige Prozesse kann man in Anlehnung an Fritzsche und Vandrei (2014: 478) auch als "negative Nachfrageschocks" bezeichnen. Im Zuge des demographischen Wandels werden sowohl sinkende Bevölkerungszahlen als auch mittelfristig steigende, aber langfristig sinkende Haushaltszahlen in Deutschland prognostiziert (Held/ Waltersbacher 2015). Vom Nachfragerückgang sind ganz unterschiedliche Bestände betroffen, meist bzw. zuerst wenig wettbewerbsfähige Häuser und Wohnungen. Diesbezüglich wird in der Literatur vor allem auf ältere Mehrfamilienhäuser mit Ausstattungs- und Modernisierungsdefiziten verwiesen (Statistische Ämter des Bundes und der Länder 2014a: 44).

Beim dauerhaften Desinvestment wird in einen Wohnungsmarkt bzw. in Teilbestände davon über einen längeren Zeitraum nicht oder viel zu wenig investiert. Die Folge davon ist eine sukzessive qualitative Verschlechterung des Wohnungsangebotes. Ab einem bestimmten Zeitpunkt fallen Wohnungen dann als Folge unterlassener Reparaturen und Instandsetzungen länger bzw. generell aus der (normalen) Nutzung, weil sie gravierende Mängel aufweisen oder schlicht unbewohnbar werden. Vom Leerstand sind hier die ältesten bzw. qualitativ schlechtesten Bestände in Bezug auf die Ausstattung am stärksten betroffen. Hierfür hat die Gebäudeund Wohnungszählung 1995 in Ostdeutschland ein eindrucksvolles Beispiel geliefert. Der massive Leerstand konzentrierte sich hier in den unsanierten, nichtmodernisierten Mehrfamilienhausbeständen der Gründerzeit (Statistische Ämter des Bundes und der Länder 2014b: 46). In diesem Fall war das dauerhafte Desinvestment eine Begleiterscheinung bzw. Folge einer verfehlten Bau- und Wohnungspolitik, wie sie für die sozialistischen Länder typisch war (vgl. für die DDR Buck 2004: 352 ff.). Dauerhaftes Desinvestment kann aber auch durch Finanzmangel verursacht, durch ungeklärte 
Eigentumsverhältnisse sowie durch die Kreditpolitik von Banken bzw. spekulativ bedingt sein. Dauerhaftes Desinvestment führt zwar eigentlich zu einer Reduktion bzw. Verknappung des Angebots, bewirkt aber einen spezifischen Leerstand, der dem Markt praktisch nicht mehr zur Verfügung steht. Dieser wird daher auch als „nicht marktaktiver Leerstand" bezeichnet.

In der Regel treten diese Prozesse getrennt voneinander bzw. bestenfalls zeitlich versetzt auf. Es können aber auch Umstände eintreten, unter denen das Zusammenwirken dieser Prozesse kaum trennscharf unterschieden werden kann. Im Ergebnis kommt es, mit räumlichen und zeitlichen Unterschieden, zu sich gegenseitig verstärkenden Tendenzen und extremen Leerständen. Paradigmatisch dafür ist der Wohnungsleerstand in Ostdeutschland, der sich schon in den 1970er- und 1980er-Jahren infolge dauerhaften Desinvestments entwickelt und die schlechte Wohnungsversorgung bzw. Wohnungsnot in der DDR verschärft hatte. In den 1990er-Jahren kamen dann zwei weitere Faktoren hinzu: eine massive Angebotsausweitung vor dem Hintergrund von Wohnungsnot und der Prognose der positiven Entwicklung der Haushaltszahlen einerseits, ein gewisser Nachfragerückgang, insbesondere in den großen Städten, andererseits. Das führte zu einer Verschränkung und gegenseitigen Verstärkung und erklärt, warum es zu einem so rasanten Umschlag von Wohnungsnot zu Überangebot kam, weshalb der Leerstand in den Großstädten derartige Ausmaße annahm und weshalb er praktisch alle Bestände eines Wohnungsmarktes betraf (vgl. Rink/Wolff/Diez 2017).

Die Ursachen und Prozesse, die zu Leerstand führen, können darüber hinaus erheblich voneinander abweichen, je nachdem, ob es sich um einen Angebotsoder Nachfragemarkt handelt. Dies kann wiederum stark von der Siedlungsstruktur abhängen und etwa zwischen städtischen, suburbanen und ländlichen Räumen variieren. Gemeinden bzw. Regionen mit Nachfrageüberhang haben meist angespannte Wohnungsmärkte mit in der Regel niedrigen Leerständen. Ein selbstständiger Prozess bzw. Leerstandsgrund ist die Umzugsmobilität der Bevölkerung, was sich in der sogenannten Leerstands- bzw. Fluktuationsreserve widerspiegelt (Buck 2004: 244). Dies kann mit der Struktur und den Charakteristika des jeweiligen Wohnungsmarktes zusammenhängen. So ist etwa in Mietermärkten die Umzugsmobilität höher als in Käufermärkten, Analoges gilt insbesondere für Universitätsstädte sowie generell für Metropolen und Großstädte im Unterschied zu Mittel- und Kleinstädten sowie ländlichen Wohnungsmärkten. Darüber hinaus ist die Wohnmobilität auch von der Bevölkerungsstruk- tur abhängig, da etwa junge Menschen öfter umziehen als Ältere und dabei andere Bestände bevorzugen als beispielsweise Familien. Dies deutet daraufhin, dass die Leerstandsstruktur in bestimmten Gebieten nur bedingt mit der jeweiligen Wohnungsbestandsstruktur zusammenhängt. Bezüglich der Lage weisen die Metropolkerne, deren Umland und andere verstädterte Regionen vor allem in Westdeutschland ein geringes Leerstandsrisiko auf (BBSR 2011: 77 ff.). Doch werden sich die Unterschiede der Nachfrageentwicklung in ihren Auswirkungen auf den Leerstand in Zukunft noch weiter räumlich ausdifferenzieren, etwa zwischen Metropolen, Mittelstädten und ländlichen Gemeinden. Insbesondere der demographische Wandel wird zukünftig für Nachfragerückgang sorgen, der jedoch durch Wanderungspräferenzen überlagert wird und daher unterschiedlich stark zutage tritt. Um diese Leerstände besser lokalisieren, spezifizieren und prognostizieren zu können, wird im Folgenden eine systematische Analyse und Typisierung vorgenommen.

\section{Methodik}

Um die Leerstandsstruktur zum Zeitpunkt der Gebäudeund Wohnungszählung $2011^{2}$ möglichst differenziert abbilden zu können, wurden die Daten für die vorliegende Studie auf der Ebene der Gemeinden analysiert. Diese kleinräumige Auswertung des Datensatzes erlaubt es, Variationen der Leerstandsstruktur zu identifizieren sowie in den bundesweiten Kontext einzuordnen.

Die Gebäude- und Wohnungszählung 2011 ist eine stichtagsbezogene Vollerhebung, wobei leerstehende Wohnungen als „am Erhebungsstichtag weder zu Wohnzwecken vermietet[e] noch von der Eigentümerin oder dem Eigentümer selbst bewohnt[e Wohnungen ...]" definiert werden (Ausnahme ist Umbau oder Modernisierung; Statistische Ämter des Bundes und der Länder 2014a: 37). Per Definition ist damit kein fluktuationsbedingter Leerstand berücksichtigt, wohl aber neu- und rückbaubedingter Leerstand (Banse/Deilmann/Fritzsche et al. 2017). Der Leerstand kann dabei in Bezug auf 50 Merkmalsausprägungen differenziert werden, die sich

2 Insgesamt erteilten 17,5 Millionen Auskunftspflichtige, das heißt Eigentümer, Verwalter und sonstige Verfügungs- und Nutzungsberechtigte, entweder postalisch oder online Auskünfte über ihre Gebäude. Die Informationen wurden auch von Wohnungsunternehmen direkt eingeholt und nur in Sonder- bzw. Ausnahmefällen, wie z. B. „fehlenden, unvollständigen oder widersprüchlichen Antworten" wurden vorher angekündigte Erhebungsbeauftragte eingesetzt (Thomsen 2011: 132). 
Tabelle 1: Formel des Regressionsmodells mitsamt berücksichtigter Merkmalsausprägungen
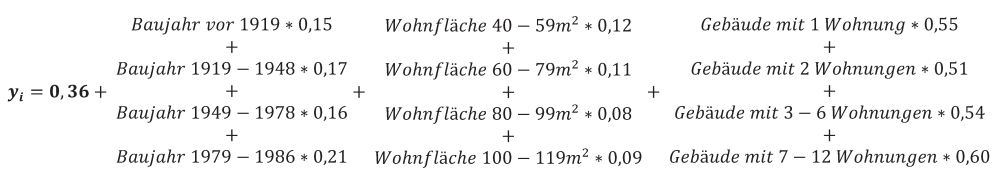

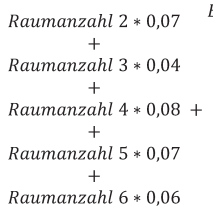

Raumanzahl $6 * 0,06$

sieben inhaltlichen Merkmalen zuordnen lassen (für eine Übersicht vgl. Statistische Ämter des Bundes und der Länder 2014b: 9 und 14 ff.): Baualter, Eigentumsform, Wohnfläche, Raumanzahl, Heizungsart, Ausstattung, Gebäudegröße. Analysiert wurden die Leerstandsquote und die Leerstandsmerkmale (absolute Anzahl von Wohnungen, jeweils insgesamt und leerstehend) in Wohngebäuden (9. Mai 2011, ohne Wohnheime), die durch das Statistische Bundesamt im Juni 2016 für alle Gemeinden mit dem Gebietstand 11. Mai 2011 zur Verfügung gestellt wurden. Für die Beantwortung der Forschungsfragen wurden drei Analyseschritte durchgeführt.

Mithilfe von Korrelationsberechnungen wurden die 50 Merkmalsausprägungen auf ihren Erklärungsgehalt für die Leerstandsquote ${ }^{3}$ auf der Bundesebene sowie für Ost- und Westdeutschland geprüft. Dabei lassen sich den leerstehenden Wohnungen für jede Merkmalsausprägung jeweils ein Korrelationskoeffizient $\left(R^{2}\right)$ zuordnen sowie ein mittlerer Anteil der Bestände der jeweiligen Merkmalsausprägungen am gesamten leerstehenden Wohnungsbestand. Die Gegenüberstellung beider Werte erlaubt Rückschlüsse darüber, in welchen Kombinationen die Merkmale zur Erklärung der Leerstandsquote beitragen und inwieweit diese Erklärung durch den jeweiligen Marktanteil zustande kommt. Dafür wurden fünf Gruppen gebildet, die im Ergebnisteil erläutert werden.

In einem zweiten Schritt wurden Gemeinden mittels eines Regressionsansatzes zu räumlichen Typen zusammengefasst, um Aufschluss über die kommunale Leerstandsstruktur in Deutschland zu erhalten. Dabei wurden hochkorrelierte Merkmalsausprägungen (mit Korrelation $>0,75^{4}$ ) sowie 101 Ausreißer ausgeschlossen, welche über die Cook-Distanz, Hebelwerte sowie fallweise Diagnose der (ausgelassen-studentisierten) Residuen identifiziert wurden. Durch die gestaffelte

3 Hierfür wurde der Anteil einer Merkmalsausprägung am Gesamtwohnungsbestand berechnet, da dieser deutlich weniger anfällig ist für hohe prozentuale Werte, welche durch niedrige Absolutwerte zustande kommen.

4 Ausprägungen „Badewanne/Dusche und WC vorhanden“ sowie „Zentralheizung".
Analyse nach $\mathrm{R}^{2}$ Klassen wurden die Merkmalsausprägungen für jeden Block schrittweise hinzugefügt, sofern sie dem F-Signifikanzwert zur Aufnahme genügen. Im Ergebnis berücksichtigt das Modell 23 Merkmalsausprägungen für insgesamt 11.256 Gemeinden (vgl. Tabelle 1).

Tabelle 2 stellt die wesentlichen Parameter für die Modellgüte des gewählten Modells dem Grundmodell gegenüber, in welches ohne methodische Vorentscheidungen alle Fälle und Merkmalsausprägungen Eingang gefunden haben. Das adjustierte Bestimmtheitsmaß $\left(R^{2}\right)$ als Hauptkriterium der Modellgüte als auch die Effektstärke, das heißt der insgesamt durch das Modell erklärte Anteil der Varianzen über alle Merkmalsausprägungen und Fälle, bescheinigt dem gewählten Modell insgesamt einen hohen Erklärungsgehalt. Dagegen sind von den 44 im Grundmodell berücksichtigten Merkmalsausprägungen nur 17 signifikant, was in der großen Streuung bestimmter Ausprägungen sowie dem starken Einfluss bestimmter Fälle, ausgedrückt im Cook-Distanz-Schwellwert, begründet ist.

Dagegen lässt der Durbin-Watson-Koeffizient, der die Residuen auf Autokorrelation testet, beim Finalmodell auf keine systematischen Zusammenhänge der Residuen und damit einer zufälligen Anordnung der Fälle über alle 23 berücksichtigten Merkmalsausprägungen schließen (vgl. Tabelle 2). Zudem unterstreichen alle Variablentoleranzen (VIF Variance Inflation Factor), dass keine der betroffenen Merkmalsausprägungen aus dem Regressionsmodell ausgeschlossen oder durch eine andere Ausprägung ersetzt werden muss und bescheinigen somit den berücksichtigten Merkmalsausprägungen in Anbetracht der hohen Fallanzahl einen hohen Erklärungsbeitrag.

In Bezug auf die berücksichtigten Fälle liegt der durch das Modell nicht erklärte Anteil (Residuen) von $82 \%$ aller Gemeinden innerhalb des Toleranzbereiches ( \pm 1 Standardabweichungen um den tatsächlichen Wert). Ebenso bescheinigen die Tests auf Normalverteilung der Residuen und Homoskedastizität als Gütemaße dem Modell in Anbetracht der hohen Anzahl an Fällen und Merkmalsausprägungen eine robuste und hohe Aussagekraft (vgl. Abbildung 1). Einzig der Test auf Linearität deutet in bestimmten Bereichen auf eine systematische 
Tabelle 2: Modellgüte des gewählten Modells („Final 4.1“) und des Grundmodells („Global 0.0“)

\begin{tabular}{|c|c|c|c|c|c|c|c|c|c|c|c|}
\hline Modell & $\begin{array}{l}\text { Merkmals- } \\
\text { ausprägung } \\
\left({ }^{* *} \text { signifikant }\right)\end{array}$ & Fälle & $\mathbf{R}$ & $\mathbf{R}^{2}$ & $\begin{array}{l}\text { Adjustiertes } \\
\text { Bestimmtheits- } \\
\text { maß } \mathbf{R}^{2}\end{array}$ & $\begin{array}{l}\text { Standard- } \\
\text { fehler }\end{array}$ & $\begin{array}{l}\text { Effekt- } \\
\text { stärke }\end{array}$ & $F$ & $\begin{array}{l}\text { Signifikanz } \\
\text { zweiseitig }\end{array}$ & $\begin{array}{l}\text { Durbin- } \\
\text { Watson }\end{array}$ & $\begin{array}{l}\text { Fälle } \\
>\text { Cook- } \\
\text { Distanz- } \\
\text { Limit } \\
\end{array}$ \\
\hline $\begin{array}{l}\text { Global } \\
0.0\end{array}$ & $44\left(17^{\star *}\right)$ & 11.339 & 0,971 & 0,943 & 0,942 & 0,724 & 4,05 & 4224,3 & 0.000 & 2,021 & 920 \\
\hline $\begin{array}{l}\text { Final } \\
4.1\end{array}$ & $23\left(22^{* *}\right)$ & 11.238 & 0,969 & 0,938 & 0,938 & 0,729 & 3,90 & 6570,3 & 0.000 & 1,977 & 587 \\
\hline
\end{tabular}

Normalverteilung

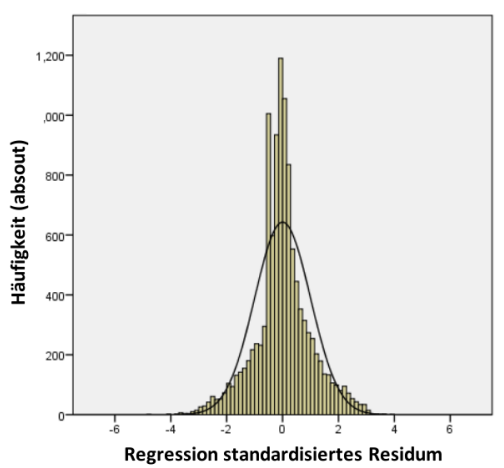

Linearität

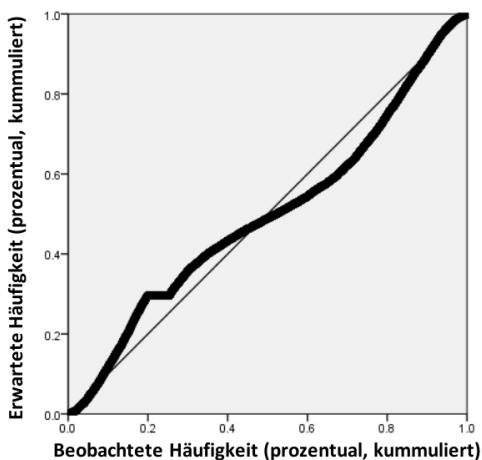

Homoskedastizität

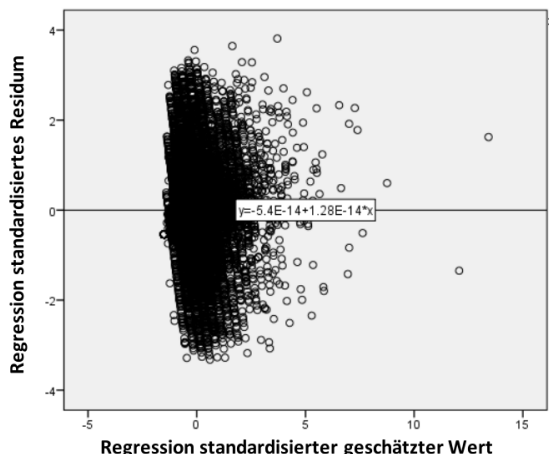

Abbildung 1: Gütemaße des gewählten Modells

Verzerrung der Residuen, was in der großen Anzahl der nicht berücksichtigten Merkmalsausprägungen begründet liegt.

Auf dieser Grundlage wurden Leerstandstypen von Gemeinden durch unterschiedliche Kombinationen der 23 Merkmalsausprägungen gebildet. Dabei wurden die Werte für jede Merkmalsausprägung in die Regressionsformel eingesetzt (vgl. Tabelle 1), die neun Ausprägungen mit dem höchsten Erklärungsgehalt ausgelesen und jedem Merkmal zugeordnet, um diese gleichermaßen zu berücksichtigen. Die Ausprägungen wurden einer schrittweisen Generalisierung innerhalb eines Merkmals unterzogen. Eine Typisierung detektierte anschließend jene 17 verbliebenen Merkmalsausprägungen, welche für das jeweilige Merkmal und den jeweiligen Fall den höchsten Erklärungsgehalt aufweisen. Im Ergebnis konnten fünf wesentliche Typen der Leerstandsstruktur identifiziert werden, welche anhand der Kombination der für sie prägenden Ausprägungen charakterisiert werden können (vgl. Tabelle 3). ${ }^{5}$

5 Darüber hinaus wurden auch Einflüsse aufgenommen, die durch nicht berücksichtigte Merkmalsausprägungen im Modell verursacht werden, was insbesondere Fälle außerhalb des Gütebereiches von
Im Zuge der Auswertung wurde die Typisierung mithilfe eines Geoinformationssystems den einzelnen Gemeinden mit Gebietsstand 31. Dezember 2011 über den amtlichen Gemeindeschlüssel zugeordnet (Geobasiskarte des Bundesamtes für Kartographie und Geodäsie mit Stand 2016) und in einer Karte visualisiert. Vor dem Hintergrund der für jeden Typ charakteristischen Kombination von Leerstandsausprägungen sowie deren räumlicher Lage wurde schließlich die Bezeichnung gewählt, wie sie Tabelle 3 zusammenfasst.

Zur Verifikation der Ergebnisse und um zu erfassen, inwieweit die Leerstandsstruktur ein Spiegelbild der Wohnungsbestandsstruktur ist, wurde die Leerstandsstruktur der resultierenden fünf Typen mit der Wohnungs-

1 Standardabweichung der Residuen betrifft. Dafür wurden alle Merkmalsausprägungen geprüft, die nicht im Modell berücksichtigt wurden (insgesamt 50-23 = 27), indem ihre Werte untereinander abgeglichen und analog ihres höchsten Erklärungsfaktors ausgelesen und gruppiert wurden. Diese Gruppierung der Residuen, das heißt der bisher nicht im Modell berücksichtigte Erklärungsgehalt, wurde über eine Kreuztabelle mit der bereits erarbeiteten Rohtypisierung verknüpft, um Umsortierungen der Fälle in andere Gruppen nach inhaltlichen Gesichtspunkten zuzulassen. 
Tabelle 3: Typisierung auf der Grundlage der Regressionsanalyse

\begin{tabular}{|c|c|c|c|c|c|c|}
\hline \multirow[t]{2}{*}{ Typ } & \multicolumn{6}{|c|}{ Hauptcharakteristik } \\
\hline & Baujahr & Gebäudegröße & Eigentumsform & Wohnfläche & Raumanzahl & Heizungsart \\
\hline \multirow[t]{4}{*}{$\begin{array}{l}\text { Leerstände kommunaler } \\
\text { Wohnungsunternehmen }\end{array}$} & $\begin{array}{l}\text { vor / nach } \\
1948\end{array}$ & Mehrfamilienhaus & $\begin{array}{l}\text { Kommune / } \\
\text { kommunales } \\
\text { Wohnungs- } \\
\text { unternehmen }\end{array}$ & unter $80 \mathrm{~m}^{2}$ & 2 bis 4 Räume & $\begin{array}{l}\text { Fernheizung } \\
\text { (Fernwärme) }\end{array}$ \\
\hline & nach 1948 & Mehrfamilienhaus & $\begin{array}{l}\text { Kommune / } \\
\text { kommunales } \\
\text { Wohnungs- } \\
\text { unternehmen }\end{array}$ & unter $80 \mathrm{~m}^{2}$ & 2 bis 4 Räume & $\begin{array}{l}\text { Fernheizung } \\
\text { (Fernwärme) }\end{array}$ \\
\hline & vor 1948 & $\begin{array}{l}\text { Ein-/Zwei- und } \\
\text { Mehrfamilienhaus }\end{array}$ & $\begin{array}{l}\text { Kommune / } \\
\text { kommunales } \\
\text { Wohnungs- } \\
\text { unternehmen }\end{array}$ & unter $80 \mathrm{~m}^{2}$ & 2 bis 4 Räume & $\begin{array}{l}\text { Einzel-/ } \\
\text { Mehrraumöfen }\end{array}$ \\
\hline & $\begin{array}{l}\text { vor / nach } \\
1948\end{array}$ & $\begin{array}{l}\text { Ein-/Zwei- } \\
\text { familienhaus }\end{array}$ & $\begin{array}{l}\text { Kommune / } \\
\text { kommunales } \\
\text { Wohnungs- } \\
\text { unternehmen }\end{array}$ & $\begin{array}{l}\text { unter } 80 \mathrm{~m}^{2} / \\
80 \mathrm{~m}^{2} \text { und mehr }\end{array}$ & $\begin{array}{l}2 \text { bis } 4 \text { Räume / } \\
5 \text { und } 6 \text { Räume }\end{array}$ & $\begin{array}{l}\text { Einzel-/ } \\
\text { Mehrraumöfen }\end{array}$ \\
\hline \multirow{3}{*}{$\begin{array}{l}\text { Leerstände in } \\
\text { Nachkriegsbeständen } \\
\text { von Ein- und } \\
\text { Mehrfamilienhäusern } \\
\text { des ländlichen und } \\
\text { suburbanen Raumes }\end{array}$} & nach 1948 & $\begin{array}{l}\text { Ein-/Zwei- } \\
\text { familienhaus }\end{array}$ & $\begin{array}{l}\text { Natürliche } \\
\text { Personen }\end{array}$ & $\begin{array}{l}\text { unter } 80 \mathrm{~m}^{2} / \\
80 \mathrm{~m}^{2} \text { und mehr }\end{array}$ & $\begin{array}{l}2 \text { bis } 4 \text { Räume / } \\
5 \text { und } 6 \text { Räume }\end{array}$ & $\begin{array}{l}\text { Fernheizung } \\
\text { (Fernwärme) }\end{array}$ \\
\hline & nach 1948 & $\begin{array}{l}\text { Ein-/Zwei- und } \\
\text { Mehrfamilienhaus }\end{array}$ & $\begin{array}{l}\text { Natürliche } \\
\text { Personen }\end{array}$ & $80 \mathrm{~m}^{2}$ und mehr & 4 bis 6 Räume & $\begin{array}{l}\text { Fernheizung } \\
\text { (Fernwärme) }\end{array}$ \\
\hline & nach 1948 & $\begin{array}{l}\text { Ein-/Zwei- und } \\
\text { Mehrfamilienhaus }\end{array}$ & $\begin{array}{l}\text { Natürliche } \\
\text { Personen }\end{array}$ & unter $80 \mathrm{~m}^{2}$ & $\begin{array}{l}2 \text { bis } 4 \text { Räume / } \\
5 \text { und } 6 \text { Räume }\end{array}$ & $\begin{array}{l}\text { Fernheizung } \\
\text { (Fernwärme) }\end{array}$ \\
\hline \multirow{3}{*}{$\begin{array}{l}\text { Leerstände in } \\
\text { Mehrfamilienhäusern des } \\
\text { Alt- und Nachkriegsbau- } \\
\text { bestandes verdichteter } \\
\text { Räume }\end{array}$} & vor 1948 & $\begin{array}{l}\text { Ein-/Zwei- und } \\
\text { Mehrfamilienhaus }\end{array}$ & $\begin{array}{l}\text { Natürliche } \\
\text { Personen }\end{array}$ & $\begin{array}{l}\text { unter } 80 \mathrm{~m}^{2} / \\
80 \mathrm{~m}^{2} \text { und mehr }\end{array}$ & $\begin{array}{l}2 \text { bis } 4 \text { Räume / } \\
5 \text { und } 6 \text { Räume }\end{array}$ & $\begin{array}{l}\text { Fernheizung } \\
\text { (Fernwärme) }\end{array}$ \\
\hline & $\begin{array}{l}\text { vor / nach } \\
1948\end{array}$ & Mehrfamilienhaus & $\begin{array}{l}\text { Natürliche } \\
\text { Personen }\end{array}$ & unter $80 \mathrm{~m}^{2}$ & 2 bis 4 Räume & \\
\hline & $\begin{array}{l}\text { vor / nach } \\
1948\end{array}$ & $\begin{array}{l}\text { Ein-/Zwei- und } \\
\text { Mehrfamilienhaus }\end{array}$ & $\begin{array}{l}\text { Natürliche } \\
\text { Personen }\end{array}$ & $\begin{array}{l}\text { unter } 80 \mathrm{~m}^{2} / \\
80 \mathrm{~m}^{2} \text { und mehr }\end{array}$ & $\begin{array}{l}2 \text { bis } 4 \text { Räume / } \\
5 \text { und } 6 \text { Räume }\end{array}$ & $\begin{array}{l}\text { Fernheizung } \\
\text { (Fernwärme) }\end{array}$ \\
\hline \multirow{4}{*}{$\begin{array}{l}\text { Leerstände im } \\
\text { Einfamilienhausbestand } \\
\text { ländlicher Räume und } \\
\text { Kleinstädte }\end{array}$} & vor 1948 & $\begin{array}{l}\text { Ein-/Zwei- } \\
\text { familienhaus }\end{array}$ & $\begin{array}{l}\text { Natürliche } \\
\text { Personen }\end{array}$ & unter $80 \mathrm{~m}^{2}$ & $\begin{array}{l}2 \text { bis } 4 \text { Räume / } \\
5 \text { und } 6 \text { Räume }\end{array}$ & \\
\hline & $\begin{array}{l}\text { vor / nach } \\
1948\end{array}$ & $\begin{array}{l}\text { Ein-/Zwei- } \\
\text { familienhaus }\end{array}$ & $\begin{array}{l}\text { Natürliche } \\
\text { Personen }\end{array}$ & unter $80 \mathrm{~m}^{2}$ & $\begin{array}{l}2 \text { bis } 4 \text { Räume / } \\
5 \text { und } 6 \text { Räume }\end{array}$ & $\begin{array}{l}\text { Fernheizung } \\
\text { (Fernwärme) }\end{array}$ \\
\hline & vor 1948 & $\begin{array}{l}\text { Ein-/Zwei- } \\
\text { familienhaus }\end{array}$ & $\begin{array}{l}\text { Natürliche } \\
\text { Personen }\end{array}$ & $80 \mathrm{~m}^{2}$ und mehr & 4 bis 6 Räume & \\
\hline & $\begin{array}{l}\text { vor / nach } \\
1948\end{array}$ & $\begin{array}{l}\text { Ein-/Zwei- } \\
\text { familienhaus }\end{array}$ & $\begin{array}{l}\text { Natürliche } \\
\text { Personen }\end{array}$ & $80 \mathrm{~m}^{2}$ und mehr & 4 bis 6 Räume & $\begin{array}{l}\text { Fernheizung } \\
\text { (Fernwärme) }\end{array}$ \\
\hline \multirow{2}{*}{$\begin{array}{l}\text { Leerstände in } \\
\text { Einfamilienhäusern mit } \\
\text { Substandard }\end{array}$} & vor 1948 & $\begin{array}{l}\text { Ein-/Zwei- } \\
\text { familienhaus }\end{array}$ & $\begin{array}{l}\text { Natürliche } \\
\text { Personen }\end{array}$ & $\begin{array}{l}\text { unter } 80 \mathrm{~m}^{2} / \\
80 \mathrm{~m}^{2} \text { und mehr }\end{array}$ & $\begin{array}{l}2 \text { bis } 4 \text { Räume / } \\
5 \text { und } 6 \text { Räume }\end{array}$ & $\begin{array}{l}\text { Einzel-/ } \\
\text { Mehrraumöfen }\end{array}$ \\
\hline & $\begin{array}{l}\text { vor / nach } \\
1948\end{array}$ & $\begin{array}{l}\text { Ein-/Zwei- } \\
\text { familienhaus }\end{array}$ & $\begin{array}{l}\text { Natürliche } \\
\text { Personen }\end{array}$ & $\begin{array}{l}\text { unter } 80 \mathrm{~m}^{2} / \\
80 \mathrm{~m}^{2} \text { und mehr }\end{array}$ & $\begin{array}{l}2 \text { bis } 4 \text { Räume / } \\
5 \text { und } 6 \text { Räume }\end{array}$ & $\begin{array}{l}\text { Einzel-/ } \\
\text { Mehrraumöfen }\end{array}$ \\
\hline
\end{tabular}


bestandsstruktur abgeglichen, indem der prozentuale Anteil jeder Ausprägung pro Merkmal für leerstehende Wohnungen mit dem Anteil für alle Wohnungen verglichen wurde.

\section{Ergebnisse}

\subsection{Einfluss von Wohnungsmerkmalen auf die Leerstandsquote}

Verschiedene Merkmale und ihre jeweiligen Ausprägungen haben einen unterschiedlichen Einfluss auf die Leerstandsquote. Auf der Bundesebene ist die Höhe der Leerstandsquote entscheidend abhängig vom Baualter, von der Gebäude- und der Wohnungsgröße. Die Korrelationsanalyse macht deutlich, dass besonders der Altbaubestand (vor 1919 errichtet) sowie die Nachkriegsbaubestände der 1950er- bis 1970er-Jahre einen besonders hohen Erklärungsgehalt für die jeweilige Leerstandsquote besitzen. Darüber hinaus sind auch Bestände der Baualtersklassen 1919-1948 sowie 1976-1986 von Leerstand betroffen, wenn auch geringer ausgeprägt als die beiden erstgenannten Baualtersklassen (vgl. Abbildung 2). In Bezug auf die Gebäudegröße haben in erster Linie die Ausprägung Mehrfamilienhaus (mit 3-6 bzw. 7-12 Wohnungen), aber auch Ein- und Zweifamilienhaus einen sehr hohen Erklärungsgehalt für die Leerstandsquote. Diese Erkenntnis deckt sich auch mit dem Einfluss der Wohnungsgröße, da bundesweit betrachtet hohe Leerstände in erster Linie Wohnungen zwischen $40 \mathrm{~m}^{2}$ und $80 \mathrm{~m}^{2}$, mit drei bis vier Räumen betreffen. Zusätzlich weisen die Merkmalsausprägungen der Eigentumsform „Privatpersonen“, das Vorhandensein einer Zentralheizung sowie die Ausstattung „Badewanne, Dusche und WC vorhanden" besonders hohe Korrelationskoeffizienten auf und einen damit vermeintlich hohen Erklärungsgehalt für die Leerstandsquote. Jedoch lässt sich anhand von Abbildung 1 deutlich ablesen, dass diese hohen Koeffizienten durch den jeweils sehr hohen mittleren Anteil von Wohnungen dieser Ausprägung am gesamten leerstehenden Wohnungsbestand zustande kommen, welche im Durchschnitt über $65 \%$ betragen.

Die Gegenüberstellung der Korrelationskoeffizienten als Maß für den Erklärungsgehalt der jeweiligen Merkmalsausprägung und dem mittleren Anteil von Wohnungen dieser Ausprägung am gesamten leerstehenden Wohnungsbestand in Abbildung 2 zeigt fünf Gruppen auf, die sich durch ähnliche Werte und inhaltliche Zusammenhänge kennzeichnen:
- Eine erste Gruppe ist geprägt durch einen sehr hohen Erklärungsgehalt für Leerstände, die sich im Altbaubestand (Baualter vor 1919) konzentrieren und Wohnungen mit drei oder vier Räumen und einer Wohnfläche zwischen $40 \mathrm{~m}^{2}$ und $60 \mathrm{~m}^{2}$ in Mehrfamilienhäusern (zwischen drei und sechs Wohnungen) umfassen. Der vergleichsweise hohe Erklärungsgehalt sowie der mittlere Marktanteil deuten auf hohe Leerstandsquoten von Wohnungen mit diesen Merkmalen hin, welche jedoch auch durch den größeren Marktanteil zustande kommen.

- Eine zweite Gruppe umfasst Leerstände kleiner Wohnungen (zwei Räume) in großen Mehrfamilienhäusern der Zwischen- und Nachkriegsjahre (Baualter 1919-1948), die sich vor allem im Bestand kommunaler Wohnungsunternehmen befinden. Der niedrige Marktanteil bei vergleichsweise hohem Erklärungsgehalt lässt auf hohe Leerstandsquoten der Wohnungen mit diesen Merkmalen schließen.

- Demgegenüber steht eine dritte Gruppe, die Einund Zweifamilienhäuser mit fünf Räumen und einer Fläche zwischen $80 \mathrm{~m}^{2}$ und $90 \mathrm{~m}^{2}$ beinhaltet, die vornehmlich zwischen 1949 und 1978 gebaut wurden. Anders als bei Gruppe 2 kommt der Erklärungsgehalt dieser Merkmale für die Leerstandsquote stärker durch deren Marktanteile zum Tragen, wozu noch die Merkmalsausprägungen einer hohen Raumanzahl (über sechs Räume), großen Wohnfläche $\left(100 \mathrm{~m}^{2}\right.$ bis $\left.140 \mathrm{~m}^{2}\right)$ im Eigentum von Gemeinschaften von Wohnungseigentümern beigefügt werden können.

- Eine vierte Gruppe weist niedrige Marktanteile und einen nur mäßig stark ausgeprägten Erklärungsgehalt für die Leerstandsquote auf. Inhaltlich ist diese Gruppe stark durchmischt, kann jedoch in drei Untergruppen gegliedert werden. Eine erste Untergruppe (4a; vgl. Abbildung 2) lässt auf Großwohnbestände in Ost- und Westdeutschland der 1970erund 1980er-Jahre schließen, welche die Merkmale Fläche $<40 \mathrm{~m}^{2}$, Baualter 1979-1986, Fernheizung, Gebäude mit über 12 Wohnungen und Zugehörigkeit zu Wohnungsgenossenschaften vereinen und deren geringeren Erklärungsgehalt für die Leerstandsquote auch in Rückbauprogrammen dieser Bestände begründet liegt. Eine zweite Untergruppe (4b) schließt sich inhaltlich Gruppe 3 an, da es sich hierbei um Bestände mit großen Wohnungen (über sechs Räume, $100 \mathrm{~m}^{2}$ bis $140 \mathrm{~m}^{2}$ ) im Eigentum von Gemeinschaften von Wohnungseigentümern in vermutlich eher ländlich geprägten Gebieten handelt. Eine dritte Untergruppe (4c) vereint die Merk- 


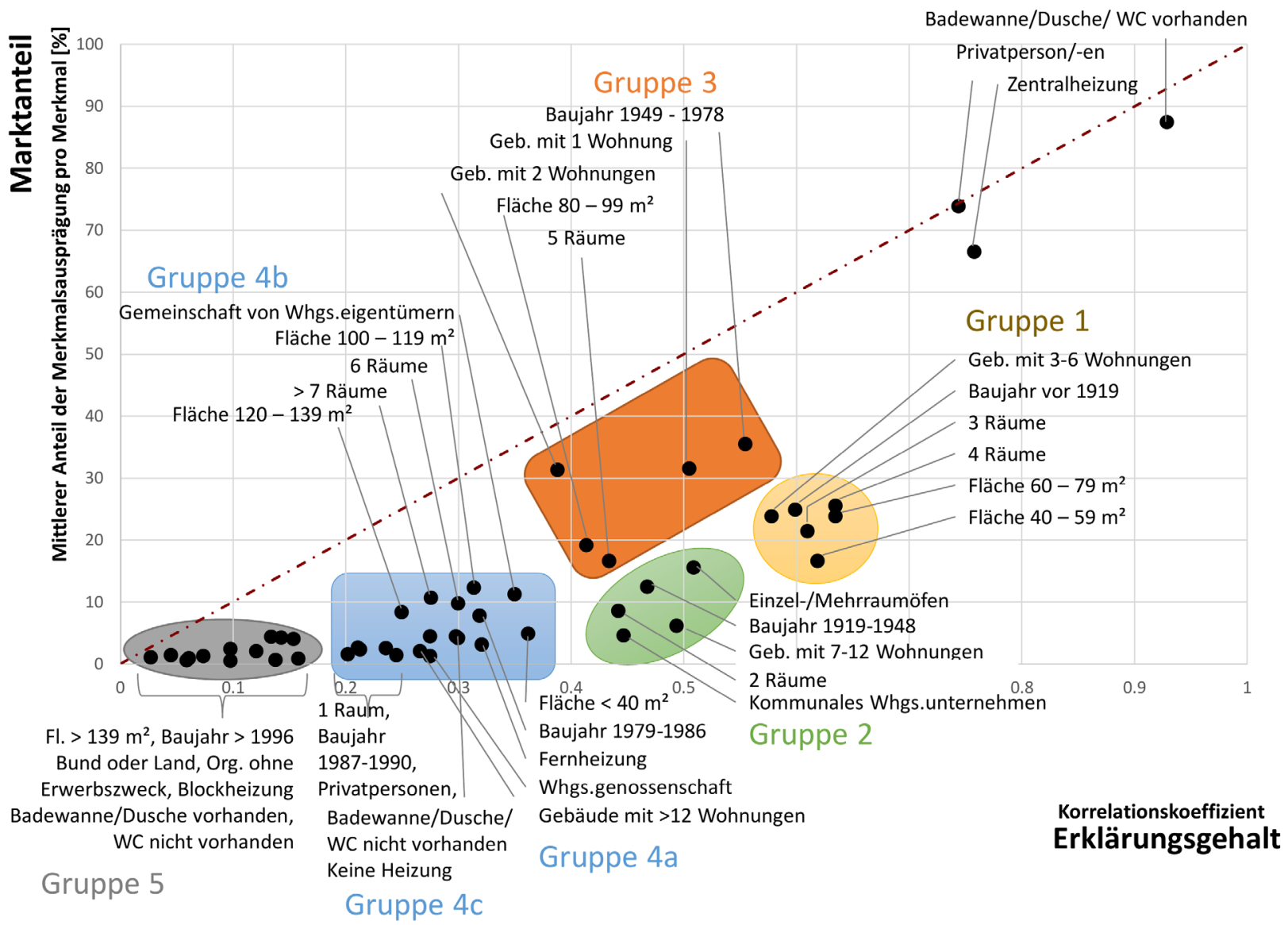

Abbildung 2: Gruppen aus unterschiedlichen Korrelationskoeffizienten der Merkmalsausprägungen zur Leerstandsquote und aus mittleren Anteilen pro Merkmalsausprägung auf Bundesebene

malsausprägung „Keine Heizung im Gebäude oder in den Wohnungen" sowie „Badewanne/Dusche und WC nicht vorhanden" und deutet damit auf nicht bezugsfähige bzw. nicht bewohnbare Bestände mit mehrheitlich einem Raum im Besitz von Privatpersonen hin.

- Schließlich ist eine fünfte Gruppe zu nennen, die sowohl geringe mittlere Anteile als auch eine geringe Erklärungskraft für die Leerstandsquote aufweist. Dies betrifft insbesondere Bestände, die nach 1996 gebaut wurden oder eine Wohnungsgröße von über $140 \mathrm{~m}^{2}$ oder einen gewissen Substandard (Blockheizung, kein WC) aufweisen oder sich im Eigentum des Bundes oder anderer Organisation ohne Erwerbszweck (z. B. Kirche) befinden.

Eine Differenzierung der Korrelationsanalyse nach Ostund Westdeutschland offenbart, wo sich prägnante Unterschiede zwischen beiden Teilen der Bundesrepublik abzeichnen. Abbildung 3 zeigt, dass in Westdeutschland vor allem die Baualtersgruppen vor 1919 und 1949-1978 einen großen Erklärungsgehalt für die Leerstandsquote besitzen - womit sowohl Altbau- als auch Nachkriegsbestände von Leerständen betroffen sind -, während dies in Ostdeutschland in erster Linie die Baualtersklasse zwischen 1949 und 1978 betrifft. Ebenso ist die Korrelation von Leerständen kommunaler Wohnungsunternehmen in Ostdeutschland sehr viel prägnanter, wie auch die Tatsache, dass in Ostdeutschland eher kleinere Wohnungen (40-60 m², 2-3 Räume) in Mehrfamilienhäusern (Gebäude mit 3-6 sowie 7-12 Wohnungen) von Leerstand betroffen sind. In Westdeutschland ist dagegen der Erklärungsgehalt größerer Wohnungen (60-80 $\mathrm{m}^{2}$ mit 4 Räumen) in Ein- und Zweifamilienhäusern deutlicher ausgeprägt.

\subsection{Räumliche Typen der Leerstands- struktur auf der Gemeindeebene}

Aufbauend auf den Ergebnissen der Korrelationsanalyse werden räumliche Leerstandstypen gebildet, 


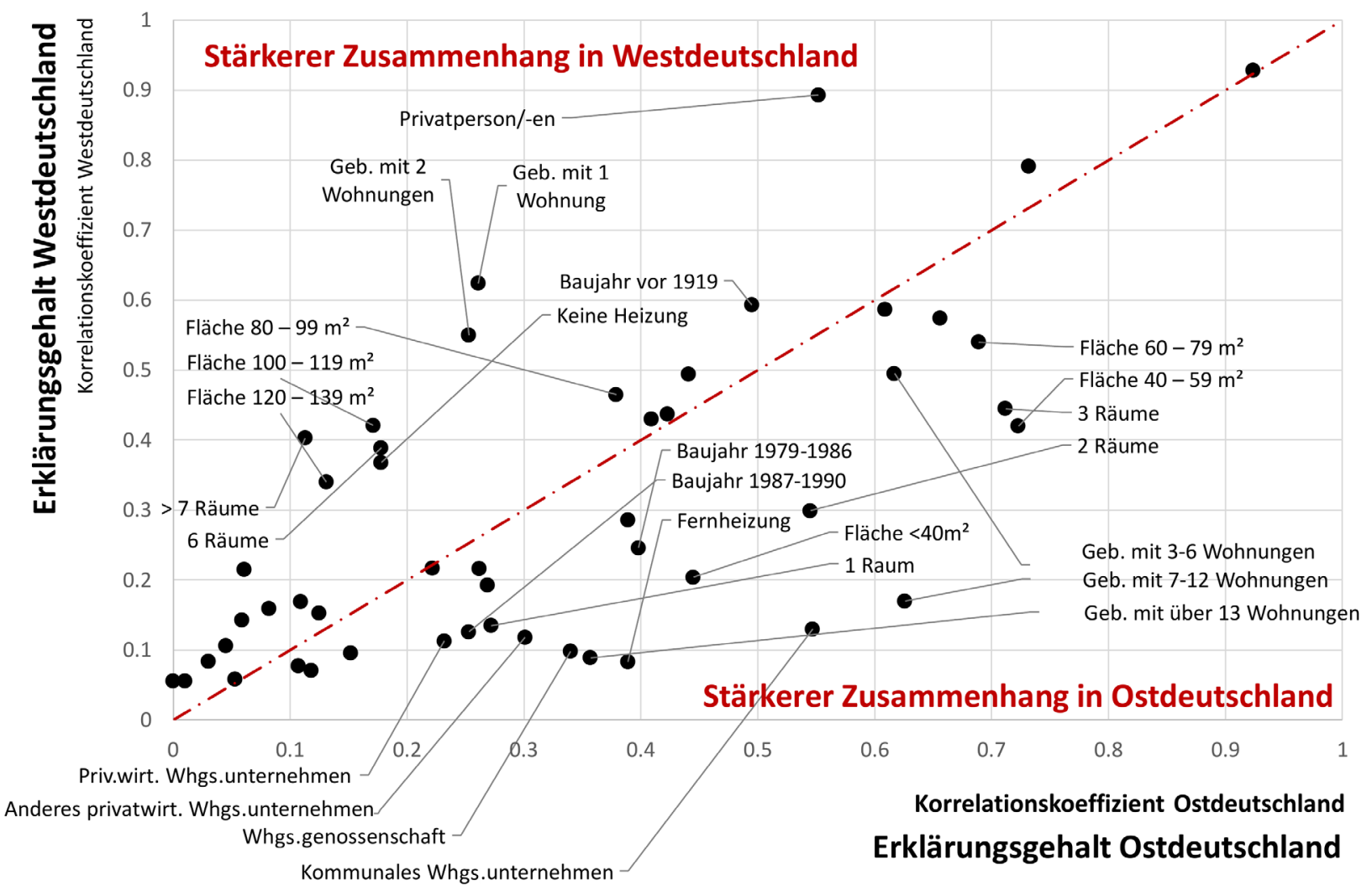

Abbildung 3: Korrelationskoeffizienten und narrative Zusammenfassung im Vergleich Ost- zu Westdeutschland

welche die Leerstandsstruktur in Deutschland auf kommunaler Ebene wiedergeben. Zu diesem Zweck wurde eine eigene Typisierung von deutschen Gemeinden zum Zeitpunkt des Zensus 2011 erarbeitet. Daraus gehen fünf unterschiedliche Typen der Leerstandsstruktur hervor (vgl. Abbildung 4), die sich in Bezug auf ihre Leerstandszusammensetzung, Lage und Größe der jeweiligen Gemeinden unterscheiden.

\subsubsection{Leerstände kommunaler Wohnungsunternehmen}

In Ostdeutschland konzentrieren sich Leerstände in den Beständen kommunaler Wohnungsunternehmen, der Anteil der Gemeinden beträgt jedoch nur $5 \%$. Die vom Leerstand betroffenen Bestände umfassen dabei vornehmlich kleine und mittelgroße Wohnungen in Gebäuden der Baualtersklasse zwischen 1949 und 1978 (zumeist DDR-Plattenbau). Darüber hinaus sind aber noch Bestände betroffen, deren Leerstand sich im Altbau (errichtet vor 1919) verschiedener Wohnungsgrößen mit zum Teil Substandard konzentriert. Gemeinden dieses Typs decken zwar nur $5 \%$ des gesamten Wohnungsmarktbestandes in Deutschland ab, jedoch auch fasst $10 \%$ des gesamten Leerstandes der Bundesrepublik, was die entsprechend hohe durchschnittliche Leerstandsquote von $8,6 \%$ bestätigt (Boxplot in Abbildung 4).

\subsubsection{Leerstände in Nachkriegsbeständen von Ein- und Mehrfamilienhäusern des ländlichen und suburbanen Raumes}

Kommunen, deren Leerstände sich in erster Linie auf den Nachkriegsbestand im Besitz von Privatpersonen (nach 1949 errichtet) beziehen und vor allem in suburbanen sowie ländlichen Regionen zu finden sind, umfassen $15 \%$ aller Gemeinden. Darunter fallen sowohl Kommunen mit Leerständen vornehmlich in Mehrfamilienhausbeständen mit vergleichsweise kleineren Wohnungen als auch in Eigenheimbeständen, das heißt Ein- und Zweifamilienhäuser mit größeren Wohnflächen und Raumanzahlen. Bereits jede fünfte Wohnung in Deutschland (20\%) kann diesem Typ zugeordnet werden, jedoch entfallen gerade mal $15 \%$ des gesamten Leerstandes auf Gemeinden dieses Typs, was eine relativ niedrige durchschnittliche Leerstandsquote von 3,4 \% ergibt. 

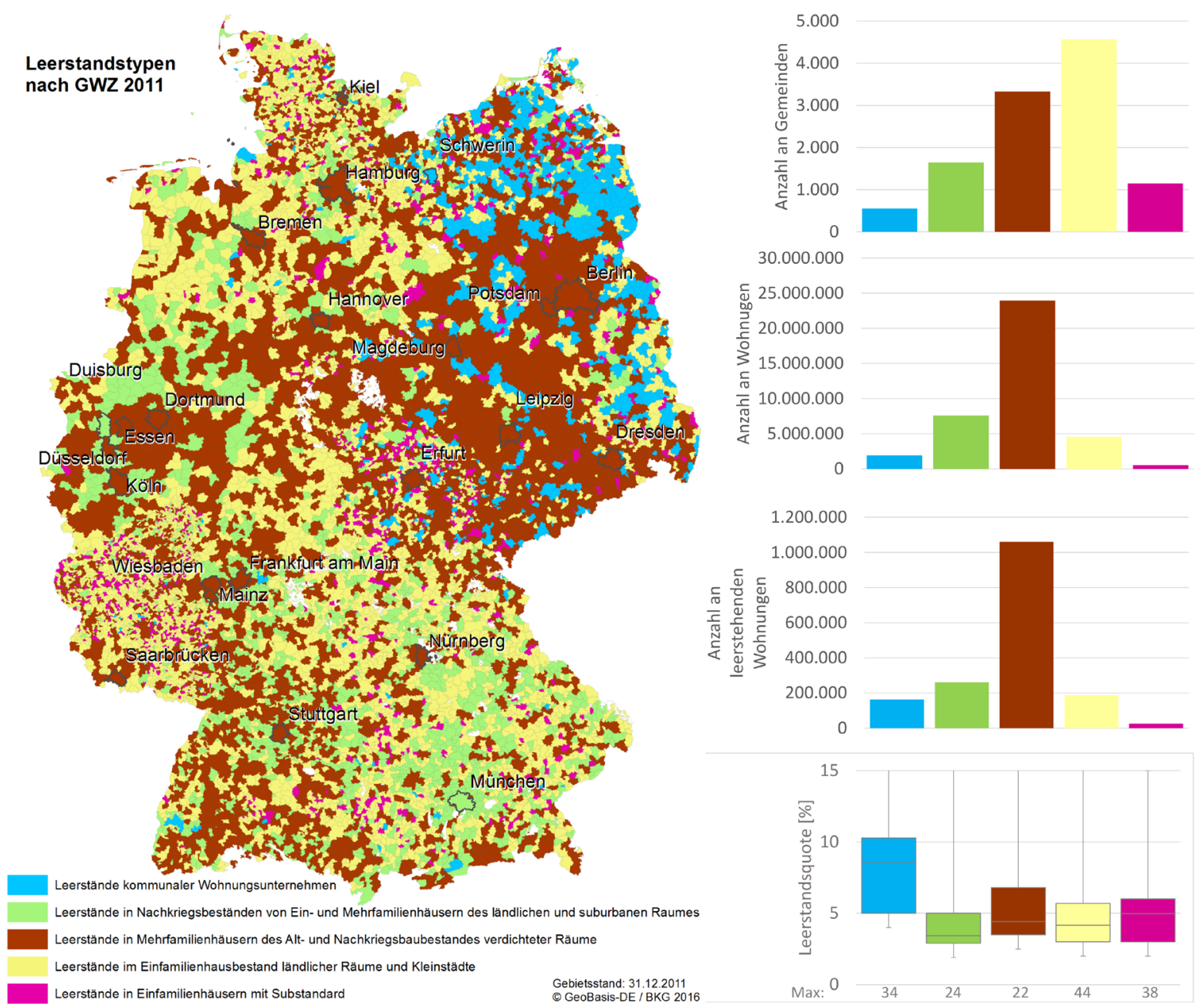

Abbildung 4: Räumliche Typen der Leerstandsstruktur auf der Gemeindeebene

\subsubsection{Leerstände in Mehrfamilienhäusern des Alt- und Nachkriegsbaubestandes verdichteter Räume}

Leerstände in verdichteten Räumen sind mehrheitlich durch Mehrfamilienhausbestände gekennzeichnet (30 \%). ${ }^{6}$ Dabei ist in erster Linie der Altbau betroffen, wozu ein substanzieller Teil ostdeutscher Gemeinden zählt und was insbesondere in dem dortigen hohen Anteil am Altbaubestand begründet liegt. Ein weitaus größerer Teil dieses Typs vereint jedoch Leerstände in Altbaubeständen des gesamten Bundesgebietes sowie im Nachkriegsbaubestand, erbaut zwischen 1949 und 1979, welche vor allem in den alten Bundesländern auftreten. Da dieser Typ in erster Linie Städte und verdichtete Räume in Deutschland abbildet, entfallen jeweils zwei

6 DiesistinersterLinieeinAusdruck derWohnungsbestandsstruktur dieses Typs: Städte weisen im Vergleich zu anderen Raumkategorien einen deutlich höheren Anteil an Mehrfamilienhäusern auf.
Drittel des gesamten Wohnungsbestandes auf diese Gemeinden sowie zwei Drittel des gesamten Bestandes leerstehender Wohnungen. Im Schnitt weist dieser Typ eine durchschnittliche Leerstandsquote von 4,4\% auf, wobei die Quoten innerhalb dieses Typs erheblich streuen (Boxplot in Abbildung 4).

\subsubsection{Leerstände im Einfamilienhausbestand ländli- cher Räume und Kleinstädte}

Ein vierter Typ kennzeichnet Leerstände ländlicher Gemeinden und Kleinstädte vor allem in Westdeutschland, welche $41 \%$ aller Kommunen ausmachen. Diese können verhältnismäßig kleine und mittelgroße Wohnungen in Zwei- und Mehrfamilienhäusern betreffen, wie sie zumeist in zentralen Ortslagen anzutreffen sind. Darüber hinaus können sich Leerstände aber auch verstärkt in großen bis sehr großen privaten Ein- bzw. Zwei- 
familienhäusern des Altbaus mit entsprechend großer Wohnfläche und Raumanzahl wie etwa Höfe konzentrieren. Dieser Typ vereint insgesamt $12 \%$ des gesamten Wohnungsbestandes sowie $11 \%$ des gesamten Leerstandes, jedoch mit einer leicht unterdurchschnittlichen Leerstandsquote von $4,2 \%$.

\subsubsection{Leerstände in Einfamilienhäusern mit Substandard}

$10 \%$ aller Gemeinden sind einem Typ zuzuordnen, dessen Leerstände vor allem auf Altbaubestände mit einer oder zwei Wohnungen sowie mit Substandard zurückzuführen sind. Hier stehen hauptsächlich Wohnungen mit Ofenheizung oder ohne Heizungsanlage leer, wobei sowohl private Ein- als auch kleinere Mehrfamilienhäuser mit drei bis sechs Wohneinheiten betroffen sind, die vor 1918 gebaut wurden. Obwohl diese Gemeinden nur jeweils $1,5 \%$ des Wohnungsbestandes in Deutschland ausmachen, weisen sie im Durchschnitt die zweithöchste Leerstandsquote von 5,0 \% auf.

\subsection{Abgleich der Leerstandsstruktur mit der Wohnungsbestandsstruktur}

Die ermittelte Leerstandsstruktur der identifizierten fünf Typen weicht zum Teil deutlich von der Struktur des jeweiligen kommunalen Wohnungsmarktes ab. Abbildung 5 stellt für jeden Typ den prozentualen Anteil jeder Merkmalsausprägung pro Merkmal für leerstehende Wohnungen dem Anteil für alle Wohnungen gegenüber (z. B. alle Baualtersklassen, die dann in der Summe $100 \%$ ergeben). Dabei wird ersichtlich, dass über alle Typen hinweg Abweichungen festzustellen sind, die vor allem bei den Merkmalen Baualter, Gebäudegröße, Eigentümerstruktur, Wohnungsgröße sowie Ausstattung besonders deutlich hervortreten.

\subsubsection{Gebäudegröße}

Gebäude mit einer Wohneinheit sind über alle räumlichen Typen hinweg nicht signifikant von Leerstand betroffen. Jedoch weicht die Leerstandsstruktur im Bereich der Zweifamilienhäuser stärker zugunsten hoher Leerstände in den Typen „Leerstände in Nachkriegsbeständen von Ein- und Mehrfamilienhäusern des ländlichen und suburbanen Raumes“ sowie „Leerstände im Einfamilienhausbestand ländlicher Räume und Kleinstädte“ ab. Interes- santerweise sind es vor allem Gebäude mit drei bis sechs Wohnungen, die in jedem Typ stark von der jeweiligen Wohnungsbestandsstruktur abweichen. Schließlich sind hohe Leerstände in Gemeinden mit „Leerständen kommunaler Wohnungsunternehmen" größeren Gebäuden mit über sieben Wohnungen zuzuordnen.

\subsubsection{Baualter}

Deutlich sind auch die Unterschiede beim Baualter, denn über alle Typen hinweg sind vor allem Altbauten, die vor 1918 errichtet wurden, von Leerstand betroffen. Demgegenüber finden sich niedrige Leerstände in Gebäuden, die nach 1996 errichtet wurden. Dies wird besonders in Gemeinden mit „Leerständen im Einfamilienhausbestand ländlicher Räume und Kleinstädte" sowie mit „Leerständen kommunaler Wohnungsunternehmen“ deutlich. In letztgenanntem Typ konzentrieren sich darüber hinaus Leerstände in der Baualtersklasse 1979 bis 1986. Im Hinblick auf den Typ „Leerstände in Nachkriegsbeständen von Ein- und Mehrfamilienhäusern des ländlichen und suburbanen Raumes" geben die Differenzen interessanterweise auch Aufschluss über stark von Leerstand betroffene Bestände, die zwischen 1949 und 1978 errichtet wurden.

\subsubsection{Eigentumsform}

Insbesondere bei den Typen „Leerstände in Nachkriegsbeständen von Ein- und Mehrfamilienhäusern des ländlichen und suburbanen Raumes" sowie „Leerstände in Mehrfamilienhäusern des Alt- und Nachkriegsbaubestandes verdichteter Räume" sind sehr geringe Leerstände vor allem bei Beständen im Besitz von Gemeinschaften von Wohnungseigentümern festzustellen, während dies beim Typ "Leerstände kommunaler Wohnungsunternehmen" vor allem Bestände von Privatpersonen betrifft. Erhöhte Leerstände können in diesen drei Typen insbesondere bei Beständen im Besitz von privatwirtschaftlichen sowie kommunalen Wohnungsunternehmen identifiziert werden, wobei letztere Eigentumsform bei Gemeinden mit „Leerständen kommunaler Wohnungsunternehmen" sehr deutlich hervortritt.

\subsubsection{Wohnungsgröße}

Die Betrachtung der Raumanzahl wie auch der Wohnfläche zeigt dagegen kaum Unterschiede zwischen den 
Typen. Es wird jedoch deutlich, dass die Leerstandsstruktur insbesondere in Wohnungen zwischen $40 \mathrm{~m}^{2}$ und $80 \mathrm{~m}^{2}$ und rund drei Räumen zugunsten hoher Leerstände abweicht. Dies ist insbesondere deutlich bei „Leerständen kommunaler Wohnungsunternehmen" sowie "Leerständen in Mehrfamilienhäusern des Alt- und Nachkriegsbaubestandes verdichteter Räume". Bei mittelgroßen (80-100 $\mathrm{m}^{2}$ ) sowie großen Wohnungen $\left(\mathrm{ab} 160 \mathrm{~m}^{2}\right.$ ) als auch bei Einraumwohnungen treten die geringsten Abweichungen zwischen Leerstands- und Wohnungsbestandsstruktur auf (vgl. Abbildung 5).

\subsubsection{Ausstattung}

Wie bereits erwähnt, ist die Heizungsart kein ausreichend differenzierendes Merkmal für die Leerstandsstruktur. Über alle Typen hinweg finden sich starke positive Abweichungen (geringer Leerstand) vor allem bei Beständen mit Zentralheizung, negative Abweichungen (hoher Leerstand) bei Beständen mit Ofenheizungen. Einzig die Merkmalsausprägung „Fernheizung“ ist für hohe Leerstände in Gemeinden mit „Leerständen kommunaler Wohnungsunternehmen" verantwortlich, die Ausprägung „Keine Heizung“ für hohe Leerstände beim Typ „Leerstände in Einfamilienhäusern mit Substandard". Ähnliches gilt für das Ausstattungsmerkmal "Bad/Dusche“. Eine fehlende Ausstattung zeigt besonders große Abweichungen beim Typ „Leerstände in Einfamilienhäusern mit Substandard“ und bei „Leerständen kommunaler Wohnungsunternehmen", während eine vorhandene Ausstattung zumeist niedrige Leerstände kennzeichnet und damit nur bedingt als differenzierendes Merkmal dienen kann.

\section{Diskussion}

Mit der hier vorgestellten Analyse liegt nun erstmals für das gesamte Bundesgebiet eine differenzierte Analyse des Leerstands im Hinblick auf seine Struktur und räumliche Verteilung vor. In Anlehnung an die zuvor formulierten Forschungsfragen möchten wir im Folgenden auf drei wesentliche Punkte eingehen.

\subsection{Einflussfaktoren der Leerstandsquote}

Bei der Betrachtung auf der Bundesebene wird deutlich, dass die Leerstandsquote in erster Linie durch das Baualter sowie die Gebäude- und Wohnungsgröße erklärt werden kann. Dabei weisen in erster Linie mittelgroße Mehrfamilienhäuser (mit 3-6 Wohnungen zwischen 40 und $60 \mathrm{~m}^{2}$ ) im Altbaubestand sowie größere Mehrfamilienhäuser (mit 7-12 Wohnungen unter $40 \mathrm{~m}^{2}$ ) der Zwischen- und Nachkriegsbestände die höchsten Leerstände auf. Dagegen kommt der Grad der Ausstattung (etwa mit Dusche/Badewanne/WC) in dieser Analyse kaum zum Tragen. Die Relevanz weiterer Merkmale zeigt sich darüber hinaus erst bei einer differenzierten Betrachtung zwischen Ost- und Westdeutschland, was unter anderem auf die unterschiedliche Geschichte beider Landesteile und damit Ursachen von Leerstand zurückzuführen ist (Effenberger/Banse 2010). Die größten Unterschiede zwischen Ost- und Westdeutschland sind bei den Merkmalen Baualter, Gebäudegröße und Eigentumsform zu beobachten. So weist das Baualter im Zusammenspiel mit der Gebäudegröße auf die starke Präsenz der Plattenbauten im ostdeutschen Leerstand hin (BMVBS 2013), was trotz Maßnahmen des Stadtumbaus vor allem durch den anhaltenden Nachfragerückgang bedingt ist (BMVBS/BBR 2006). Dies wird ebenso bei der Wohnungsgröße deutlich, da in Ostdeutschland eher kleine Wohnungen leerstehen. Im Gegensatz zu Westdeutschland sind in Ostdeutschland vor allem kommunale Wohnungsunternehmen von hohem Leerstand betroffen. Dagegen sind Altbaubestände der Gründerzeit, der 1950er- und 1960er-Jahre, aber auch einzelne Bestände von Großwohnsiedlungen, die durch Desinvestment immer noch nichtmarktaktiv sind (BMVBS 2013: 76) sowie Bestände mit Ausstattungsdefiziten oder baulichen Mängeln, in beiden Landesteilen gleichermaßen anzutreffen.

\subsection{Typisierung der kommunalen Leerstandsstruktur}

Für eine räumlich differenzierte Betrachtung wurden die wesentlichen Merkmalsausprägungen auf Gemeindebasis mittels multivariater Statistik zu fünf räumlichen Typen zusammengefasst. Dabei grenzen sich die Typen dahingehend voneinander $a b$, dass die verschiedenen Merkmale unterschiedlich stark ausgeprägt sind und damit die für den Leerstand verantwortlichen Bestände anzeigen. Analog zu den in der ersten Frage identifizierten Unterschieden zwischen Ost- und Westdeutschland werden in der räumlichen Darstellung bestimmte Typen herausgestellt, die jeweils einen der beiden Landesteile charakterisieren (vgl. Effenberger 2012). So sind Gemeinden mit Leerständen kommunaler Wohnungsunternehmen vorzugsweise in Ostdeutschland, Gemein- 


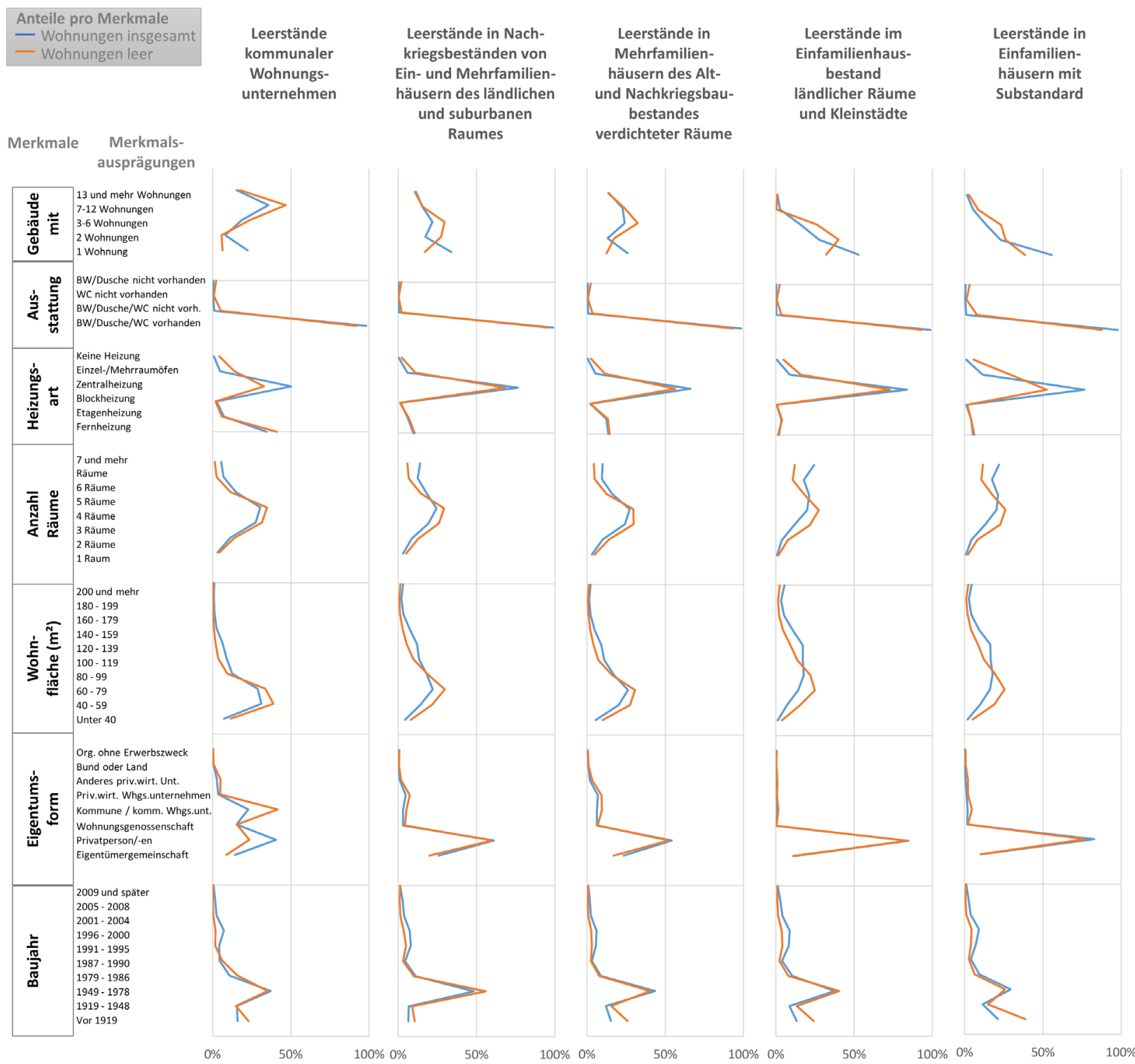

Abbildung 5: Anteile der Wohnungen (insgesamt, leer) pro Merkmal, differenziert nach Typen der Leerstandsstruktur

den mit Leerständen in Nachkriegsbeständen von Ein- und Mehrfamilienhäusern des ländlichen und suburbanen Raumes in erster Linie in Westdeutschland anzutreffen (vgl. Lerbs/Neubacher/Teske 2017). Es existieren jedoch auch Typen, die im gesamten Bundesgebiet vorkommen. Darüber hinaus spiegeln die Typen auch die unterschiedliche Siedlungsstruktur wider, sodass einige Typen in erster Linie Städte, andere vor allem suburbane Räume und weitere eher ländliche Räume charakterisieren. Die Typisierung bestätigt in ihrer kleinräumigen Darstellung, dass Leerstände in vielen ländlichen Räumen und Kleinstädten vor allem große Wohnungen sowie Einfamilienhäuser betreffen (Lohse/Vandrei 2016; Banse/Deilmann/Fritzsche et al. 2017).
Darüber hinaus macht die gezeigte Typisierung deutlich, inwieweit die Bedeutung der Erklärungsfaktoren, wie sie zu Beginn des Ergebnisteils für das Bundesgebiet analysiert wurden, in den Gemeinden räumlich variiert. Es sei erwähnt, dass auch die hier vorgestellte Typisierung eine Generalisierung der eigentlichen Situation darstellt und eher als eine Orientierung der dominanten Leerstandsstruktur zu begreifen ist, die einen Datenbestand von 50 Merkmalsausprägungen für über 11.000 Gemeinden durch fünf Typen abbildet, womit einige lokale Spezifika überdeckt werden. So können durchaus unterschiedliche Leerstandsstrukturen innerhalb einer Gemeinde auftreten. Innerhalb des Typs „Leerstände in Mehrfamilienhäusern des Alt- und Nachkriegsbau- 
bestandes verdichteter Räume" können sich etwa auch sanierte Altbaubestände befinden, im Typ „Leerstände kommunaler Wohnungsunternehmen" können durchaus auch Einfamilienhäuser leer stehen. Aufgrund günstiger Finanzierungsmöglichkeiten werden im ländlichen Raum Neubauten anstelle der Altbausubstanz bevorzugt (Deschermeier/Henger/Seipelt et al. 2017).

\subsection{Beziehung der Leerstands- zur Wohnungsbestandsstruktur}

Die Gegenüberstellung von Leerstands- und Wohnungsbestandsstruktur fügt der zuvor vorgestellten Typisierung und der damit einhergehenden Generalisierung eine zusätzliche Erklärung hinzu. Dabei wird deutlich, bei welchen Merkmalsausprägungen beide Strukturen die größten Unterschiede aufweisen. Dafür wurden die Merkmale der einzelnen Typen dahingehend geprüft, inwieweit sie die Struktur des Wohnungsbestandes widerspiegeln, um darüber Rückschlüsse auf die Höhe des Leerstandes zu ziehen. Dabei zeigt sich, dass die Leerstandsstruktur insbesondere bei den Typen „Leerstände kommunaler Wohnungsunternehmen“, „Leerstände im Einfamilienhausbestand ländlicher Räume und Kleinstädte" sowie "Leerstände in Einfamilienhäusern mit Substandard" von der Wohnungsbestandsstruktur abweicht. Die Leerstandsstruktur ist insbesondere bei Mehrfamilienhäusern kommunaler Wohnungsunternehmen mit drei bis sehs Wohnungen, die vor 1918 errichtet wurden, sowie mit sieben bis zwölf Wohnungen, die zwischen 1979 und 1986 erbaut wurden (DDR-Plattenbaubestände) besonders stark ausgeprägt (Effenberger/Banse 2010: 43). Bei Gemeinden mit Leerständen durch Substandard, wie etwa fehlendem Bad oder dem Vorhandensein von Ofenheizungen, sind ebenso große Abweichungen von der Wohnungsbestandsstruktur festzustellen. Das betrifft insbesondere Mehrfamilienhausbestände mit Wohnungen mittlerer Größe (3-4 Räume, 40-80 m²), die vor 1918 errichtet wurden. Bei Gemeinden mit „Leerständen im Einfamilienhausbestand ländlicher Räume und Kleinstädte" sind Abweichungen hauptsächlich bei kleineren Mehrfamilienhausbeständen (mit 3-6 Wohnungen) sowie Zweifamilienhausbeständen, die vor 1918 sowie zwischen 1919 und 1948 errichtet wurden, mit tendenziell größeren Wohnungen (60-100 $\mathrm{m}^{2}$ ) zu finden. Im Gegensatz dazu können auch Merkmale identifiziert werden, die auf eine sehr geringe Leerstandsquote schließen lassen. So ist der Leerstand besonders in Beständen mit einer Wohnung, Zentralheizung, über sechs Räumen, einer Wohnfläche von über $100 \mathrm{~m}^{2}$ und
Ausstattung mit Badewanne/Dusche/WC, die nach 1996 errichtet wurden, besonders niedrig. Die Dominanz der Einfamilienhausbestände ist besonders bei den Typen "Leerstände im Einfamilienhausbestand ländlicher Räume und Kleinstädte" in den zwischen 1990 und 2000 errichteten Beständen ausgeprägt.

Die Koexistenz hoher Leerstände in einigen Wohnungsbeständen und niedriger Leerstände in anderen in ein und derselben Gemeinde weist auf die unterschiedliche Wettbewerbsfähigkeit verschiedener Wohnungsbestände in den entsprechenden Märkten hin (vgl. Spehl 2011). Dahingehend ergeben sich Hinweise auf das mögliche Zusammenwirken unterschiedlicher Leerstandsursachen. Eine darauf aufbauende Analyse der Dynamik von Haushaltszahlen, Sanierungstätigkeiten oder aber Gebäudezu- und -abgängen kann Aufschluss darüber geben, inwieweit die Unterschiede zwischen Leerstands- und Wohnungsbestandsstruktur auf Nachfragerückgang, Desinvestment und Angebotsausweitung zurückzuführen ist.

\section{Fazit}

Insgesamt sind die Leerstandsdaten der Gebäude- und Wohnungszählung (GWZ) 2011 sehr robust und damit gut geeignet, um flächendeckend und kleinräumig Strukturen des Leerstandes abzubilden. Der vorliegende Beitrag nimmt erstmalig eine räumliche Typisierung der Leerstandsstrukturen deutscher Gemeinden vor. Es wird deutlich, dass einige Merkmale nur wenig zur Erklärung der Leerstandsquote beitragen, was in erster Linie für die Merkmale Ausstattung mit Badewanne/Dusche/WC sowie Heizungsart zutrifft. Dies liegt zum einen in der nur geringen Varianz der Merkmalsausprägungen sowie an dem sehr hohen Grad an Vollausstattung mit Badewanne/Dusche/WC sowohl bei bewohnten als auch leeren Wohnungen. Bezüglich der derzeit in Vorbereitung befindlichen Gebäude- und Wohnungszählung 2021 wäre in diesem Zusammenhang eine alternative Aufschlüsselung der Merkmale wünschenswert, um auf den Zustand der jeweiligen Wohnungsbestände schließen zu können. Dabei ist die Analyse der Dauer sowie der Ursachen der erfassten Leerstände zielführend, wie sie bereits in der GWZ 1987 in den alten und der GWZ 1995 in den neuen Bundesländern erfragt wurden. In Bezug auf die Dauer ist ein kurzfristiger Leerstand von bis zu drei Monaten periodisch und weist auf einen sogenannten Fluktuationsleerstand hin (Glatter 2003). Längerer Leerstand bis zu sechs Monaten kennzeichnet dagegen 
eine problematische, von mehr als sechs Monaten eine dauerhafte bzw. strukturelle Leerstandssituation. Diese Differenzierung nach lang-, mittel- und kurzfristigem Leerstand kann wiederum mit den Ursachen des Leerstandes verknüpft werden (Klebsch 1997: 536). Während langfristige Leerstände etwa die mangelnde Marktgängigkeit von Wohnungen oder Vermietungsschwierigkeiten andeuten, können kurzfristige Leerstände auf einen geplanten Verkauf bzw. Vermietung des Hauses oder der Wohnung zurückzuführen sein. Die Eigentümer könnten dahingehend befragt werden, ob ihre Wohnung oder ihr Haus in der nahen Zukunft dem Markt wieder zugeführt werden soll (vgl. Klupp/Töpper 2010: 28). Dagegen deuten bauliche Mängel oder Maßnahmen wie etwa Abriss/Rückbau bzw. Instandsetzung/Modernisierung eher auf nichtmarktaktive Leerstände hin. Nicht nur für die Wohnungsmarktforschung, sondern auch für wohnungspolitische und immobilienwirtschaftliche Entscheidungen und Investitionen erscheint die Erfassung und Kombination dieser Merkmale durchaus gewinnbringend, um zwischen am Wohnungsmarkt angebotenen und damit marktaktivem und aus diversen Gründen nichtmarktaktivem Leerstand differenzieren zu können.

Die vorgestellte Untersuchung versteht sich als raumanalytischer Beitrag, der einerseits Bündel von Einflussfaktoren für Leerstand identifiziert und andererseits die Vielfalt der in Deutschland zum Zeitpunkt der Gebäude- und Wohnungszählung (GWZ) 2011 auftretenden Leerstandsstrukturen den Gemeinden und damit kleinräumig zuordnet. Dabei liefert die Verknüpfung einer bundesweiten Korrelationsanalyse mit einer kleinräumigen Typisierung einen Beitrag zur anwendungsorientierten und evidenzbasierten Wohnungsmarktforschung im Besonderen als auch Raumforschung im Allgemeinen. Die Analyse ermöglicht es, die Raum- bzw. Wohnungsmarktbeobachtung auf der Bundesebene im Einklang mit anderen Heuristiken etwa zur Leerstandshöhe (Rink/ Wolff 2017) weiter zu qualifizieren, indem Kommunen hinsichtlich ihrer Leerstandsstruktur differenziert werden und gleichzeitig in den Bundestrend eingeordnet werden können. Dies ermöglicht es, Leerstandsstrukturen kleinräumig zu differenzieren, zu bewerten, einzuordnen und abzuleiten. Es eröffnen sich weiterführende Analysemöglichkeiten etwa in Bezug auf künftige Leerstandsrisiken. Vor dem Hintergrund unterschiedlicher Bevölkerungsdynamiken, divergierender Marktentwicklungen und gesellschaftlicher Makrotrends (neue Lebensformen, Haushaltsverkleinerungen) ist eine evidenzbasierte Wohnungsmarktanalyse und -prognostik, die auf ein flächendeckendes Monitoring von Leerstandsstrukturen und -dynamiken basiert, erforderlich. Schließlich können die Ergebnisse dazu dienen, die Raumplanung als wohnungspolitisches Instrument weiter zu qualifizieren, indem Bedarfe (etwa Neubau aber auch Abriss) ermittelt und Leerstandsrisiken abgeschätzt werden. Daraus lassen sich dann nicht nur Aussagen zum Einsatz von städtebaulichen bzw. wohnungspolitischen Maßnahmen ableiten, sondern auch die Raum- und Wohnungsmarktbeobachtung auf der Bundesebene weiter qualifizieren.

\section{Literatur}

Banse, J.; Deilmann, C.; Fritzsche, C.; Hörnig, V.; Kluge, J.; Kretzschmar, D.; Marquardt, G.; Motzek, T.; Ragnitz, J.; Thum, M.; Vandrei, L. (2017): Auswirkungen der demografischen Entwicklung auf den ostdeutschen Wohnungsmarkt. Dresden. = ifo Dresden Studien 78.

BBSR - Bundesinstitut für Bau-, Stadt- und Raumforschung (2011): Wohnungs- und Immobilienmärkte in Deutschland 2011. Bonn. $=$ Analysen Bau.Stadt.Raum 5 .

BBSR - Bundesinstitut für Bau-, Stadt und Raumforschung (2014): Aktuelle und zukünftige Entwicklung von Wohnungsleerständen in den Teilräumen Deutschlands, Bonn.

BMVBS - Bundesministerium für Verkehr, Bau und Stadtentwicklung (2013): Bericht über die Wohnungs- und Immobilienwirtschaft in Deutschland. Berlin.

BMVBS - Bundesministerium für Verkehr, Bau und Stadtentwicklung; BBR - Bundesamt für Bauwesen und Raumordnung (2006): Stadtumbau Ost - Stand und Perspektiven. Erster Statusbericht der Bundestransferstelle. Berlin.

BMVBS - Bundesministerium für Verkehr, Bau und Stadtentwicklung; BBR - Bundesamt für Bauwesen und Raumordnung (Hrsg.) (2007): Grundstückswertermittlung im Stadtumbau. Verkehrswertermittlung bei Schrumpfung und Leerstand. Bonn. = Forschungen 127.

Buck, H. F. (2004): Mit hohem Anspruch gescheitert - Die Wohnungspolitik der DDR. Münster.

Deschermeier, P.; Henger, R.; Seipelt, B.; Voigtländer, M. (2017): Wohnungsmangel in den Städten, Leerstand auf dem Land. Köln. = IW-Kurzberichte 44.2017.

Effenberger, K.-H. (2012): Wohnungsleerstand heute: Im Westen sind es 2,5 Mio., im Osten 1 Mio. In: Wohnungswirtschaft heute $45,15-17$.

Effenberger, K.-H.; Banse, J. (2010): Städtische Wohnungsbestände in Ostdeutschland im Vergleich. Stuttgart.

Fritzsche, C.; Vandrei, L. (2014): Keiner will sie haben - Theoretische Ursachen für Immobilienleerstand. In: Credit and Capital Markets - Kredit und Kapital 47, 3, 465-483. doi: 10.3790/ ccm.47.3.465

Glatter, J. (2003): Strategien der Wohnungsunternehmen in schrumpfenden und wachsenden Märkten. In: Hutter, G.; Iwanow, I.; Müller, B. (Hrsg.) (2003): Demographischer Wandel und Strategien der Bestandsentwicklung in Städten und Regionen. Dresden, 149-172. = IÖR-Schriften 41.

Gutting, R.; Vandrei, L. (2018): Wohnungsleerstand - ein großes Problem für kleine Kommunen? In: ifo Dresden berichtet 25 , 2, 7-11. 
Held, T.; Waltersbacher, M. (2015): Wohnungsmarktprognose 2030. Bonn. = BBSR-Analysen KOMPAKT 07/2015.

Henger, R.; Schier, M.; Voigtländer, M. (2013): Wohnungsleerstand. Eine wirtschaftspolitische Herausforderung. Köln. = IW-Positionen 62.

Hoekstra, J.; Vakili-Zad, C. (2011): High Vacancy Rates and Rising House Prices: The Spanish Paradox. In: Tijdschrift voor economische en sociale geografie 102, 1, 55-71. doi: 10.1111/j.14679663.2009.00582.x

Kitchin, R.; O'Callaghan, C.; Gleeson, J. (2014): The New Ruins of Ireland? Unfinished Estates in the Post-Celtic Tiger Era. In: International Journal of Urban and Regional Research 38, 3, 1069-1080. doi: 10.1111/1468-2427.12118

Klebsch, O. (1997): Leerstand von Wohnungen. In: Mändle, E.; Galonska, J. (Hrsg.): Wohnungs- und Immobilien-Lexikon. Hamburg, 536-537.

Klupp, M.; Töpper, J. (2010): Eigentümerbefragung zum Wohnungsleerstand. Endbericht. Hamburg. https://digital.zlb.de/viewer/ rest/image/15479692/IBB_Leerstandsanalyse_2010.pdf/full/ max/0/IBB_Leerstandsanalyse_2010.pdf (18.12.2018).

Kreibich, V. (1985): Wohnversorgung und Wohnstandortverhalten. In: Friedrichs, J. (Hrsg.): Die Städte in den 80er Jahren. Demographische, ökonomische und technologische Entwicklungen. Opladen, 181-195.

Lerbs, O.; Neubacher, J.; Teske, M. (2017): Wohnungsleerstand in Ostdeutschland: Begünstigende Faktoren, kleinräumige Trends und Auswirkungen auf Marktwerte. In: ifo Dresden berichtet 24, 2, 12-20.

Lohse, J.; Vandrei, L. (2016): Leerstand von Einfamilienhäusern: Ein dörfliches Problem? In: ifo Dresden berichtet 23, 6, 20-25.

Pfeiffer, U.; Simons, H.; Porsch, L. (2001): Wohnungswirtschaftlicher Strukturwandel in den neuen Bundesländern. Bericht der Kommission im Auftrag des Bundesministeriums für Verkehr, Bau- und Wohnungswesen. Stuttgart.

Rink, D.; Wolff, M. (2015): Wohnungsleerstand in Deutschland. Zur Konzeptualisierung der Leerstandsquote als Schlüsselindikator der Wohnungsmarktbeobachtung anhand der GWZ 2011. In: Raumforschung und Raumordnung 73, 5, 311-325. doi: 10.1007/s13147-015-0361-8

Rink, D.; Wolff, M. (2017): Wohnungsleerstand in Deutschland. Zur Qualifizierung der Leerstandsquote am Beispiel der GWZ 2011. In: BBSR - Bundesinstitut für Bau-, Stadt- und Raumforschung (Hrsg.): Lücken in der Leerstandsforschung - Wie Leerstände besser erhoben werden können. Bonn, 5-8. = BBSR Berichte KOMPAKT 02/2017.

Rink, D.; Wolff, M.; Diez, B. (2017): Wohnungsleerstand zwischen Schrumpfung und Wachstum. Eine Analyse anhand der GWZ 2011. In: Wüstenrot-Stiftung (Hrsg.): Fokus Wohnungsleerstand. Ausmaß, Wahrnehmung, kommunale Reaktionen. Darmstadt, 276-285.

Spehl, H. (Hrsg.) (2011): Leerstand von Wohngebäuden in ländlichen Räumen. Beispiele ausgewählter Gemeinden der Länder Hessen, Rheinland-Pfalz und Saarland. Hannover. = E-Paper der ARL 12.

Statistische Ämter des Bundes und der Länder (2014a): Gebäude und Wohnungsbestand in Deutschland. Erste Ergebnisse der Gebäude- und Wohnungszählung 2011. Hannover.

Statistische Ämter des Bundes und der Länder (2014b): Gebäudeund Wohnungen in den neuen Bundesländern und Berlin-Ost.
Vergleich der Ergebnisse der Gebäude- und Wohnungszählungen 2011 und 1995. Kamenz.

Thomsen, M. (2011): Zensus 2011: Gebäude- und Wohnungszählung. In: Statistische Monatshefte Niedersachsen 65, 3, 130133.

Vaché, M. (2016): Gespaltener Wohnungsmarkt: Das Beispiel der Region Frankfurt/Main. In: von Einem, E. (Hrsg.): Wohnen. Markt in Schieflage - Politik in Not. Wiesbaden, 71-98. doi: 10.1007/978-3-658-11757-3

Voigtländer, M.; Demary, M.; Gans, P.; Meng, R.; Schmitz Veltin, A.; Westerheide, P. (2009): Wirtschaftsfaktor Immobilien. Die Immobilienmärkte aus gesamtwirtschaftlicher Perspektive. Im Auftrag des Deutschen Verbandes für Wohnungswesen, Städtebau und Raumordnung und der Gesellschaft für Immobilienwirtschaftliche Forschung. Berlin/Wiesbaden. 